\title{
On embeddings of circulant graphs
}

\author{
Marston Conder \\ Department of Mathematics \\ University of Auckland \\ Auckland 1142, New Zealand \\ m. conder@auckland.ac.nz
}

\author{
Ricardo Grande \\ Faculty of Science and Technology \\ University of the Basque Country (UPV/EHU) \\ 48080 Bilbao, Spain \\ rgrande001@ikasle.ehu.es
}

Submitted: Jan 10, 2014; Accepted: May 9, 2015; Published: May 22, 2015

Mathematics Subject Classifications: 05C10 (primary), 05E18, 20B25, 57M15

\begin{abstract}
A circulant of order $n$ is a Cayley graph for the cyclic group $\mathbb{Z}_{n}$, and as such, admits a transitive action of $\mathbb{Z}_{n}$ on its vertices. This paper concerns 2-cell embeddings of connected circulants on closed orientable surfaces. Embeddings on the sphere (the planar case) were classified by Heuberger (2003), and by a theorem of Thomassen (1991), there are only finitely many vertex-transitive graphs with minimum genus $g$, for any given integer $g \geqslant 3$. Here we completely determine all connected circulants with minimum genus 1 or 2 ; this corrects and extends an attempted classification of all toroidal circulants by Costa, Strapasson, Alves and Carlos (2010).
\end{abstract}

\section{Introduction}

A circulant is a Cayley graph for a cyclic group. To be more precise, if $n$ is any positive integer and $X$ is any subset of $\mathbb{Z}_{n} \backslash\{0\}=\{1,2, \ldots, n-1\}$, then the circulant $C_{n}(X)$ is the undirected simple graph of order $n$ with vertex-set $\mathbb{Z}_{n}=\{0, \ldots, n-1\}$ and edge-set $\left\{\{i, i+a\}: i \in \mathbb{Z}_{n}, a \in X\right\}$. Under addition $\bmod n$, the group $\mathbb{Z}_{n}$ induces a regular group of automorphisms of $C_{n}(X)$; for example, the mapping $i \mapsto i+1(\bmod n)$ gives an automorphism that permutes the $n$ vertices of $C_{n}(X)$ in a cycle: $(0,1,2, \ldots, n-1)$. Hence in particular, every circulant $C_{n}(X)$ is vertex-transitive, and therefore regular, of valency $k=|X \cup(-X)|=\mid\left\{x \in \mathbb{Z}_{n}:(x \in X)\right.$ or $\left.(-x \in X)\right\}$.

Examples include the 2-valent simple cycle $C_{n}$ (with $X=\{1\}$ ), and the $(n-1)$-valent complete graph $K_{n}$ (with $X=\mathbb{Z}_{n} \backslash\{0\}$ ). Note that the valency $k$ can be even or odd, but is odd if and only if $n$ is even and $X$ contains $\frac{n}{2}$.

If $X=\left\{a_{1}, a_{2}, \ldots, a_{m}\right\}$, then we also denote $C_{n}(X)$ by $C_{n}\left(a_{1}, a_{2}, \ldots, a_{m}\right)$. By the above observations, we may assume that $0<a_{1}<a_{2}<\cdots<a_{m} \leqslant \frac{n}{2}$.

It is well known (and easy to see) that the circulant $C_{n}\left(a_{1}, \ldots, a_{m}\right)$ is connected if and only if $\operatorname{gcd}\left(a_{1}, \ldots, a_{m}, n\right)=1$. More generally, the number of connected components of 
$C_{n}\left(a_{1}, \ldots, a_{m}\right)$ is $d=\operatorname{gcd}\left(a_{1}, \ldots, a_{m}, n\right)$, with each of the vertices $0,1, \ldots, d-1$ lying in different components, and with each component being isomorphic to $C_{n / d}\left(a_{1} / d, \ldots, a_{m} / d\right)$.

Two circulant graphs $C_{n}(X)=C_{n}\left(a_{1}, \ldots, a_{m}\right)$ and $C_{n}(Y)=C_{n}\left(\tilde{a}_{1}, \ldots, \tilde{a}_{m}\right)$ of the same order are said to satisfy Ádám's relation if there exists an integer $r$ coprime to $n$ such that $Y \equiv r X \bmod n$, that is, $\left\{\tilde{a}_{1}, \tilde{a}_{2}, \ldots, \tilde{a}_{m}\right\}=\left\{r a_{1}, r a_{2}, \ldots, r a_{m}\right\}$ as subsets of $\mathbb{Z}_{n}$. When this happens, $C_{n}(X)$ is isomorphic to $C_{n}(Y)$, with multiplication by the unit $r$ (mod $n$ ) giving an isomorphism. Conversely, it can happen that two circulants are isomorphic without Ádám's relation being satisfied; for example, this occurs with $C_{16}(1,2,7)$ and $C_{16}(2,3,5)$.

Various properties of circulants (and their adjacency matrices, which are circulant matrices) are well known, for example with regard to chromatic number, connectivity, domination properties, factorisations, metric dimension, self-complementarity, and symmetry.

Also a number of things are known about the embeddability of connected circulants on surfaces. All of them are upper-embeddable, which means that they all have an embedding on an orientable surface (of maximum possible genus), with just one or two faces.

This is trivial for valency 2 (simple cycles), and for valency greater than 3 it follows from a more general theorem of Škoviera and Nedela about upper-embeddability of connected finite Cayley graphs; see [11, Proposition 7]. For valency 3, it follows from Theorem 5 in [11]), since up to isomorphism the only 3-valent connected circulants of girth 3 are $C_{4}(1,2) \cong K_{4}$ and the triangular prism graph $C_{6}(2,3)$, both of which are upper-embeddable.

The most common question about embeddability is the minimum genus of a graph: the smallest genus of all the orientable surfaces on which the graph has a 2-cell embedding. Circulant graphs that have minimum genus 0 (or equivalently, are planar) were completely classified in 2003 by Heuberger [7]:

Proposition 1. Let $C_{n}(X)=C_{n}\left(a_{1}, a_{2}, \ldots, a_{m}\right)$ be a connected circulant with $0<a_{1}<$ $a_{2}<\cdots<a_{m} \leqslant \frac{n}{2}$. Then $C_{n}(X)$ is planar if and only if one of the following holds:

- $m=1$ and $a_{1}$ is a unit in $\mathbb{Z}_{n}$, or

- $m=2$ and $n \equiv 2 \bmod 4$ and $a_{1}$ is even and $a_{2}=\frac{n}{2}$, or

- $m=2$ and $n$ is even and either $a_{2}= \pm 2 a_{1}$ or $a_{1}= \pm 2 a_{2}$ in $\mathbb{Z}_{n}$.

Equivalently, every planar connected circulant graph is isomorphic to either the simple $n$-cycle $C_{n}(1)$, or the $n$-prism $C_{n}\left(2, \frac{n}{2}\right)$ where $n \equiv 2 \bmod 4$, or $C_{n}(1,2)$ where $n$ is even.

In a major piece of work [14], Thomassen proved a conjecture of Babai, that for every $g \geqslant 3$, there are only finitely many vertex-transitive graphs with minimum genus $g$.

Recently Strapasson, Costa and Alves [12] announced a classification of circulants embeddable on the torus, but unfortunately they made some errors and omitted many cases.

In this paper, we determine up to isomorphism all the connected circulants having minimum genus 1 or 2 . In doing this, we correct and extend the results of [12], by proving the following: 
Theorem 2. A connected circulant has minimum genus 1 if and only if it is isomorphic to one or more of

- $C_{n}\left(a_{1}, a_{2}\right)$ for some $a_{1}$ and $a_{2}$ not satisfying the conditions of Proposition 1 , or

- $C_{n}\left(a_{1}, a_{2}, a_{3}\right)$ where $a_{3} \equiv \pm\left(a_{1}+a_{2}\right) \bmod n$, or

- $C_{n}\left(1,2, \frac{n}{2}\right)$, where $n \equiv 2 \bmod 4$ and $n \geqslant 6$, or

- $C_{n}\left(1,2, \frac{n}{2}-1\right)$, where $n \equiv 0 \bmod 4$ and $n \geqslant 8$, or

- $C_{8}(1,2,4)$ or $C_{9}(1,2,4)$.

Theorem 3. A connected circulant has minimum genus 2 if and only if it is isomorphic to one of

- $C_{n}\left(1,2, \frac{n}{2}\right)$, where $n \equiv 0 \bmod 4$ and $n \geqslant 12$, or

- $C_{n}\left(1,2, \frac{n}{2}-1\right)$, where $n \equiv 2 \bmod 4$ and $n \geqslant 10$, or

- $C_{n}\left(2,4, \frac{n}{2}\right)$, where $n \equiv 2 \bmod 4$ and $n \geqslant 10$, or

- $C_{12}(1,2,4), C_{12}(1,3,6), C_{12}(1,4,6), C_{12}(2,3,6), C_{12}(3,4,6), C_{8}(1,2,3,4)$ or $C_{12}(1,4,5,6)$.

In Section 2 we give some further background, and in particular, we show that if a connected circulant has minimum genus 1 or 2 , then its valency must be $3,4,5,6$ or 7 . In Section 3 we exhibit embeddings of genus 1 and 2 for the circulants listed in Theorems 2 and 3 , and then to establish those theorems, we consider circulants with valencies 7,5 and 6 in Sections 4, 5 and 6, respectively. Some of the more challenging cases are left to Section 7, where we give explanations of how they can be treated (using a range of approaches), rather than giving full details.

\section{Further background}

A 2-cell embedding of a connected graph $G$ on an orientable surface $S$ is a representation (or drawing) of $G$ on $S$ with the property that when the graph is removed from the surface, it breaks it up into simply-connected regions (homeomorphic to disks), called faces.

This creates a map, and if we denote the number of faces, edges and vertices of the map by $F, E$, and $V$ respectively, then by the well known Euler-Poincaré formula we have

$$
V-E+F=\chi=2-2 g
$$

where $\chi$ is the Euler characteristic of the surface $S$. If $g=0$ (and $\chi=2$ ) then the map is called planar or spherical, while if $g=1$ (and $\chi=0$ ) then it is Euclidean or toroidal, and if $g>1$ (and $\chi<0$ ) then the map is hyperbolic.

A connected graph $G$ can have several different embeddings, and the genus $g$ of each one is determined by the number of faces, since the numbers of vertices and edges are fixed (for given $G$ ). The smallest and largest achievable values of $g$ are called the minimum genus (or simply the genus) of $G$ and the maximum genus of $G$, respectively. The minimum genus $\gamma(G)$ occurs when the number of faces is maximised. 
Before continuing, we will adapt notation a little, and define $V(G)$ and $E(G)$ to be the vertex-set and edge-set of $G$, and $V(M), E(M)$ and $F(M)$ to be the vertex-set, edge-set and face-set of the map $M$ resulting from an embedding of $G$. (Note that $V(M)=V(G)$ and $E(M)=E(G)$ for all such $M$, but $F(M)$ is variable.)

As explained in [2] and [6] for example, every embedding of a graph $G$ on an orientable surface is uniquely determined by the cyclic orientations of the edges at the vertices of $G$. To make things easier, we will assume the graph $G$ is simple.

Then for each vertex $v$ the embedding defines a cyclic permutation $\rho_{v}$ of the neighbours of $v$, which indicates (by convention) the anti-clockwise ordering of the edges incident with $v$ when the map is viewed from the 'outside' of the surface. The set $\left\{\rho_{v}: v \in V(G)\right\}$ is then called a rotation system. Conversely, any such set of cyclic permutations gives rise to a unique embedding, and so there is a one-to-one correspondence between the orientable 2-cell embeddings of a simple graph $G$ and its rotation systems.

Moreover, the rotation system for an embedding can be used to calculate the number of faces, using the well known (and obvious) face tracing algorithm: from any vertex $u$, take an edge to one of its neighbours $v$, then 'turn left' at $v$ (using the rotation $\rho_{v}$ ) and continue to the next vertex, and so on. This process traces out a closed walk beginning with the arc $(u, v)$, and by finiteness must return at some stage to the vertex $u$ along the arc $\left(\rho_{u}^{-1}(v), u\right)$. Each such closed walk traces a face of the map, and all faces can be found by successively choosing the initial arc $(u, v)$ from among those not already used.

(Alternatively, one may 'turn right' at every vertex, instead of turning left, and in that case the same faces will be found, but with each face traced in reverse order.)

Note that if the vertex $v$ has valency $k$, then there are $(k-1)$ ! possibilities for the rotation $\rho_{v}$, and hence if $G$ has order $n$ and is regular of valency $k$, then the number of possible rotation systems is $((k-1) !)^{n}$. For small $n$ (and $k$ ), these can be enumerated by computer, but for large $k$ that becomes infeasible. Accordingly, determining the minimum genus of a given graph $G$ is computationally challenging.

In fact, the question of deciding whether a given connected graph $G$ has minimum genus $\gamma(G) \leqslant g$ (for given $g$ ) has received considerable attention. This is known as the graph genus problem, and it is computationally challenging. In [5], Filotti, Miller and Reif described an algorithm for finding an embedding of a given connected graph of order $n$ on an orientable surface of genus $g$, when such an embedding exists. This algorithm runs in $n^{O(g)}$ steps. But then it was shown by Thomassen [13] that the graph genus problem is NP-complete. More generally, finding the minimum genus is NP-hard.

Nevertheless, it is possible to determine the minimum genus of all graphs in particular families; for example, Ringel and Youngs showed that the minimum genus of the complete graph $K_{n}$ is $\left\lceil\frac{(n-3)(n-4)}{12}\right\rceil$, as a key to their proof of the Heawood Map Colouring Problem [10]. Also Ringel [9] showed earlier that the minimum genus of the complete bipartite graph $K_{m, n}$ is $\left\lfloor\frac{(m-2)(n-2)+3}{4}\right\rfloor$, which is also equal to $\left\lceil\frac{(m-2)(n-2)}{4}\right\rceil$. But even for some small graphs, determining the minimum genus can be difficult; for example, it took several pages to do this for the Cartesian product $C_{3} \times C_{3} \times C_{3}$ (on 27 vertices); see [4] (and $[8]$ ).

For circulants, the graph genus problem appears to be just as challenging as it does 
for connected graphs in general. As noted earlier, the connected circulants with minimum genus 0 were completely determined by Heuberger [7], and an attempt to classify those with minimum genus 1 was made by Strapasson, Costa and Alves [12]. In the latter paper, it was shown that some of the circulants listed in Theorem 2 have toroidal embeddings and therefore minimum genus 1 , but the authors claimed (erroneously) that the cases they gave in their Proposition 5 are the only such cases - and also they did not provide a complete proof in the 3 -valent cases.

When we attempted a classification of connected circulants with minimum genus 2 , we discovered that the graph $C_{9}(1,2,4)$ has minimum genus 1 , but did not occur in the list given in [12]. Then also we found another infinite family of other examples with minimum genus 1 , namely the circulants $C_{n}\left(a_{1}, 2 a_{1}, \frac{n}{2}-a_{1}\right)$ where $n \equiv 0 \bmod 4$ and $n \geqslant 12$; this gives the family in the fourth case of Theorem 2. It turns out there was a flaw in an argument in the proof of Proposition 4 in [12], where it was assumed that the restriction of a 6 -valent toroidal embedding to a 4-valent subgraph had quadrangular faces.

Here we note that it is easy to prove that all connected circulants $C_{n}\left(a_{1}, \ldots, a_{m}\right)$ with $m=1$ or 2 (and hence valency 2,3 or 4 ) have minimum genus 0 or 1 .

In the other direction, we have the following:

Lemma 4. All connected circulants with minimum genus 1 or 2 have valency at most 7 .

Proof. Let $G$ be any connected circulant, of order $n$ and valency $k$, such that $G$ has an orientable embedding with genus $g \leqslant 2$. Then by the Euler-Poincaré formula

$$
n-n k / 2+F=V-E+F=2-2 g \geqslant-2,
$$

where $F$ is the number of faces. Also because each face has 3 or more edges (and each edge lies in at most 2 faces), we have $3 F \leqslant 2 E=n k$, and by the inequality above

$$
2 \geqslant n k / 2-n-F \geqslant n k / 2-n-n k / 3=n(k-6) / 6 .
$$

Thus $k(k-6)<n(k-6) \leqslant 12$, and it follows that $k<8$.

Hence for minimum genus 1 or 2 we need only consider the cases where the valency $k$ is 7 (and $m=4$ ) or 5 or 6 (and $m=3$ ), as we do in Sections 4 to 6. The sporadic cases dealt with in Section 7 have minimum genus 2 or more, and for some those we give only a short description of how that can be proved. Also in one case we make use of theorems of Battle, Harary, Kodama and Youngs (see [1, Theorem 1 \& corollaries]), which show that if a graph $G$ has a subgraph that is the union of two subgraphs $H$ and $K$ (with no vertex in common), then the minimum genus of $G$ is at least equal to the sum of the minimum genera of $H$ and $K$.

\section{Some (minimal) embeddings}

In this Section, we turn to minimum genus, and begin by proving that the circulants listed in Theorems 2 and 3 have embeddings of genus 1 and 2 respectively. Note that some of 
this was done for the genus 1 case in [12], but certainly not all of it; in particular, the 6 -valent family $C_{n}\left(1,2, \frac{n}{2}-1\right)$ and the 5 -valent graph $C_{9}(1,2,4)$ were missed in Proposition 5 of [12], and also the 5 -valent cases of $C_{n}\left(a_{1}, a_{2}, a_{1}+a_{2}\right)$ were not properly considered.

Proposition 5. The circulants listed in Theorem 2 all have toroidal embeddings, and hence minimum genus 1 .

Proof. We treat each case in turn, showing that it has an orientable embedding of genus 1 , and noting that none of the circulants listed in the statement of the theorem is isomorphic to one of those in Heuberger's classification of connected planar circulants.

- $C_{n}\left(a_{1}, a_{2}\right)$ where $a_{1}$ and $a_{2}$ do not satisfy the conditions of Proposition 1 :

As explained in [12], in the 4-valent case where $0<a_{1}<a_{2}<\frac{n}{2}$, a genus 1 embedding of $C_{n}\left(a_{1}, a_{2}\right)$ can be obtained from a quadrangular map on the torus with faces of size 4 bounded by closed walks of the form $\left(i, i+a_{1}, i+a_{1}+a_{2}, i+a_{2}\right)$, or equivalently, by taking the rotation $\left(i+a_{1}, i+a_{2}, i-a_{1}, i-a_{2}\right)$ at each vertex $i$.

The 3 -valent case where $0<a_{1}<a_{2}=\frac{n}{2}$ (which was not treated properly in [12]) can be dealt with by the same argument: simply double each edge of the form $\left\{j, j+\frac{n}{2}\right\}$, then embed the resulting multigraph on the torus taking a rotation of the form $\left(i+a_{1}, i+\right.$ $\left.\frac{n}{2}, i-a_{1}, i+\frac{n}{2}\right)$ at each vertex $i$, and delete one edge from each pair of edges of the form $\left\{j, j+\frac{n}{2}\right\}$. If $\frac{n}{2}$ and $a_{1}$ are both odd, then one such toroidal embedding has rotation of the form $\left(i+a_{1}, i-a_{1}, i+\frac{n}{2}\right)$ at vertex $i$ for all even $i$, and the inverse of this for all odd $i$, and all faces have length 6 . In other cases, the face lengths can vary, with average 6 .

- $C_{n}\left(a_{1}, a_{2}, a_{3}\right)$ with $a_{3}= \pm\left(a_{1}+a_{2}\right)$ :

If $\frac{n}{2} \notin\left\{a_{1}, a_{2}, a_{3}\right\}$, then the valency is 6 , and as explained in [12], we can take a toroidal embedding of $C_{n}\left(a_{1}, a_{2}\right)$ with $n$ faces of size 4 bounded by closed walks of the form $\left(i, i+a_{1}, i+a_{1}+a_{2}, i+a_{2}\right)$, with rotation $\rho_{i}$ at each vertex $i$ being $\left(i+a_{1}, i+a_{2}, i-a_{1}, i-a_{2}\right)$, and then add the edges $\left\{i, i+a_{1}+a_{2}\right\}$ as diagonals of those quadrangular faces, to give an embedding of $C_{n}\left(a_{1}, a_{2}, a_{1}+a_{2}\right)$ in the torus with $2 n$ triangular faces. The rotation $\rho_{i}$ at each vertex $i$ is then $\left(i+a_{1}, i+a_{1}+a_{2}, i+a_{2}, i-a_{1}, i-a_{1}-a_{2}, i-a_{2}\right)$.

On the other hand, if $\frac{n}{2} \in\left\{a_{1}, a_{2}, a_{3}\right\}$ then the valency is 5 , and by rearrangement and negation if necessary we can assume that $a_{1}+a_{2}=a_{3}=\frac{n}{2}$. This case is more tricky, and was not treated properly in [12].

If $\frac{n}{2}$ is odd, we can add an edge from vertex $i$ to vertex $i+\frac{n}{2}$ as a diagonal across the quadrangular face of the toroidal embedding of $C_{n}\left(a_{1}, a_{2}\right)$ bounded by $\left(i, i+a_{1}, i+\frac{n}{2}, i+a_{2}\right)$ whenever $i$ is even; then the rotation at a vertex $i$ is $\alpha_{i}=\left(i+a_{1}, i+\frac{n}{2}, i+a_{2}, i-a_{1}, i-a_{2}\right)$ if $i$ is even, and $\beta_{i}=\left(i+a_{1}, i+a_{2}, i-a_{1}, i+\frac{n}{2}, i-a_{2}\right)$ if $i$ is odd.

Similarly, if $\frac{n}{2}$ is even, we can add the diagonal whenever $i \in\left\{0,1, \ldots, \frac{n}{2}-1\right\}$, and then we have the rotation $\alpha_{i}$ for $i \in\left\{0,1, \ldots, \frac{n}{2}-1\right\}$ and rotation $\beta_{i}$ for $i \in\left\{\frac{n}{2}, \frac{n}{2}+1, \ldots, n-1\right\}$. In both of these two sub-cases, exactly half of the $n$ quadrangular faces of the toroidal embedding of $C_{n}\left(a_{1}, a_{2}\right)$ are split into triangular faces, while the other half are unchanged, so we have $n$ triangular faces and $\frac{n}{2}$ quadrangular faces, giving a total of $\frac{3 n}{2}$, as required. 
- $C_{n}\left(1,2, \frac{n}{2}\right)$, where $n \equiv 2 \bmod 4$ and $n \geqslant 10$ :

Here a toroidal embedding can be obtained by taking the rotation $\rho_{i}$ at vertex $i$ as $\left(i+1, i-1, i-2, i+\frac{n}{2}, i+2\right)$ when $i$ is even, and $\left(i+1, i+2, i+\frac{n}{2}, i-2, i-1\right)$ when $i$ is odd. Then just as in the 5 -valent case above, we have $n$ triangular faces and $\frac{n}{2}$ quadrangular faces. The triangular faces are bounded by 3 -cycles of the form $(i, i+1, i-1)$ and $(i, i+2, i+1)$ for all even $i$, and the quadrangular faces by 4-cycles of the form $\left(i, i-2, i+\frac{n}{2}-2, i+\frac{n}{2}\right)$ for all even $i$. This is the same as the embedding described in a different way in [12].

- $C_{n}\left(1,2, \frac{n}{2}-1\right)$, where $n \equiv 0 \bmod 4$ and $n \geqslant 12$ :

In this case, we can take $\rho_{i}=\left(i+1, i+2, i+\frac{n}{2}+1, i+\frac{n}{2}-1, i-2, i-1\right)$ when $i$ is even, and its inverse $\left(i+1, i-1, i-2, i+\frac{n}{2}-1, i+\frac{n}{2}+1, i+2\right)$ when $i$ is odd. Then we have $2 n$ triangular faces, bounded by 3 -cycles of the form $(i, i+1, i+2),(i, i-1, i+1),\left(i, i+2, i+1+\frac{n}{2}\right)$ and $\left(i, i+1-\frac{n}{2}, i-1+\frac{n}{2}\right)$, for each even value of $i$. (Note that if we restrict this embedding to the 4-valent subgraph $C_{n}(1,2)$, there are faces of length $\frac{n}{2}$, bounded by the cycles $(0,2,4,6 \ldots,-4,-2)$ and $(-1,-3,-5, \ldots, 5,3,1)$. In particular, these are not quadrangular, which helps explain why this case was missed by Strapasson et al in [12],)

- $C_{8}(1,2,4)$ :

Take $\rho_{0}=(1,2,4,6,7), \rho_{1}=(2,0,5,3,7), \rho_{2}=(3,4,0,1,6), \rho_{3}=(4,2,5,7,1)$, and their analogues $\rho_{4}=(5,6,0,2,3), \rho_{5}=(6,4,1,7,3), \rho_{6}=(7,0,4,5,2), \rho_{7}=(0,6,1,3,5)$, obtainable by adding the appropriate integer mod 8 to the points in the rotation, and get a toroidal map with 8 triangular and 4 quadrangular faces (not the same as the one in [12]).

- $C_{9}(1,2,4)$ :

Take $\rho_{0}=(1,2,4,8,7,5), \rho_{1}=(2,0,5,6,8,3), \rho_{2}=(3,7,6,4,0,1)$, and their analogues $\rho_{3}=(4,5,7,2,1,8), \rho_{4}=(5,3,8,0,2,6), \rho_{5}=(6,1,0,7,3,4)$, and $\rho_{6}=(7,8,1,5,4,2)$, $\rho_{7}=(8,6,2,3,5,0), \rho_{8}=(0,4,3,1,6,7)$, and get a toroidal map with 18 triangular faces, bounded by 3 -cycles of the form $(i, i+1, i+2),(i, i-1, i-2),(i, i+2, i+4),(i, i-2, i-4)$, $(i, i+4, i-1)$ and $(i, i-4, i+1)$, for $i \in\{0,3,6\}$.

Proposition 6. The circulants listed in Theorem 3 all have embeddings on orientable surfaces of genus 2 .

Proof. Again we treat the cases in turn, but this time with not so much detail, leaving calculation of the faces to the reader.

- $C_{n}\left(1,2, \frac{n}{2}\right)$, where $n \equiv 0 \bmod 4$ and $n \geqslant 12$ :

Take $\rho_{i}= \begin{cases}\left(i+1, i-1, i-2, i+\frac{n}{2}, i+2\right) & \text { for } i \in\left\{0,2, \ldots, \frac{n}{2}-2\right\} \cup\left\{\frac{n}{2}+1, \frac{n}{2}+3, \ldots, n-3\right\} \\ \left(i+1, i+2, i+\frac{n}{2}, i-2, i-1\right) & \text { for } i \in\left\{1,3, \ldots, \frac{n}{2}-1\right\} \cup\left\{\frac{n}{2}+2, \frac{n}{2}+4, \ldots, n-2\right\} \\ \left(i+1, i+2, i+\frac{n}{2}, i-1, i-2\right) & \text { for } i=\frac{n}{2} \\ \left(i+1, i+2, i-1, i-2, i+\frac{n}{2}\right) & \text { for } i=n-1 .\end{cases}$

These rotations give an embedding with $\frac{3 n}{2}-2$ faces, of which $n-2$ are triangular, $\frac{n}{2}-2$ are quadrangular, and two have length 7 . The Euler characteristic is $n-\frac{5 n}{2}+\left(\frac{3 n}{2}-2\right)=-2$. 
- $C_{n}\left(1,2, \frac{n}{2}-1\right)$, where $n \equiv 2 \bmod 4$ and $n \geqslant 10$ :

Take $\rho_{i}= \begin{cases}\left(i+1, i+2, i+\frac{n}{2}+1, i+\frac{n}{2}-1, i-2, i-1\right) & \text { for } i \in\left\{1,3, \ldots, \frac{n}{2}-2\right\} \\ \left(i+1, i-1, i-2, i+\frac{n}{2}-1, i+\frac{n}{2}+1, i+2\right) & \text { for } i \in\left\{2,4, \ldots, \frac{n}{2}-1\right\} \\ \left(i+1, i+2, i+\frac{n}{2}+1, i+\frac{n}{2}-1, i-2, i-1\right) & \text { for } i \in\left\{\frac{n}{2}+1, \frac{n}{2}+3, \ldots, n-2\right\} \\ \left(i+1, i-1, i-2, i+\frac{n}{2}-1, i+\frac{n}{2}+1, i+2\right) & \text { for } i \in\left\{\frac{n}{2}+2, \frac{n}{2}+4, \ldots, n-1\right\} \\ \left(i+1, i+\frac{n}{2}-1, i-2, i-1, i+\frac{n}{2}+1, i+2\right) & \text { for } i=0 \text { and } \frac{n}{2} .\end{cases}$

These rotations give an embedding with $2 n-2$ faces, of which $2 n-4$ are triangular and two have length 6 . The Euler characteristic is $n-3 n+(2 n-2)=-2$.

- $C_{n}\left(2,4, \frac{n}{2}\right)$, where $n \equiv 2 \bmod 4$ and $n \geqslant 10$ :

In this case we let $n=4 k+2$ and consider two sub-cases: $k$ even, and $k$ odd.

If $k$ is even, take

$$
\rho_{i}=\left\{\begin{array}{l}
\left(i+2, i+4, i+\frac{n}{2}, i-4, i-2\right) \text { when } i \in\{1,2,5,6,9,10, \ldots, 2 k-3,2 k-2\} \\
\quad \text { or } i \in\{2 k+1,2 k+4,2 k+5,2 k+8,2 k+9, \ldots, n-6, n-5, n-2\} \\
\left(i+2, i-2, i-4, i+\frac{n}{2}, i+4\right) \quad \text { when } i \in\{0,3,4,7,8,11,12, \ldots, 2 k-1,2 k\} \\
\quad \text { or } i \in\{2 k+3,2 k+6,2 k+7,2 k+10,2 k+11, \ldots, n-4, n-3\} \\
\left(i+2, i-4, i-2, i+\frac{n}{2}, i+4\right) \text { when } i=2 k+2 \\
\left(i+2, i+\frac{n}{2}, i-4, i-2, i+4\right) \text { when } i=n-1 .
\end{array}\right.
$$

If $k$ is odd, take

$$
\rho_{i}=\left\{\begin{array}{l}
\left(i+2, i+4, i+\frac{n}{2}, i-4, i-2\right) \text { when } i \in\{1,2,5,6,9,10, \ldots, 2 k-5,2 k-4,2 k-1,2 k\} \\
\quad \text { or } i \in\{2 k+4,2 k+5,2 k+8,2 k+9, \ldots, n-8, n-7, n-4, n-3\} \\
\left(i+2, i-2, i-4, i+\frac{n}{2}, i+4\right) \text { when } i \in\{3,4,7,8,11,12, \ldots, 2 k-3,2 k-2\} \\
\quad \text { or } i \in\{2 k+1,2 k+2,2 k+6,2 k+7,2 k+10,2 k+11, \ldots, n-6, n-5, n-2, n-1\} \\
\left(i+2, i-4, i-2, i+\frac{n}{2}, i+4\right) \quad \text { when } i=2 k+3 \\
\left(i+2, i+\frac{n}{2}, i-4, i-2, i+4\right) \text { when } i=0 .
\end{array}\right.
$$

In both sub-cases, again the rotations give an embedding with $\frac{3 n}{2}-2$ faces, of which $n-2$ are triangular, $\frac{n}{2}-2$ are quadrangular, and two have length 7 , and the Euler characteristic is $n-\frac{5 n}{2}+\left(\frac{3 n}{2}-2\right)=-2$.

- $C_{12}(1,2,4)$

Take $\rho_{i}= \begin{cases}(i+1, i+2, i-4, i+4, i-2, i-1) & \text { for } i \text { even } \\ (i+1, i-1, i-2, i+4, i-4, i+2) & \text { for } i \text { odd. }\end{cases}$

These rotations give an embedding with 22 faces, of which 16 are triangular and 6 are quadrangular, giving Euler characteristic $12-36+22=-2$.

- $C_{12}(1,3,6)$

There seems to be no 'nice' genus 2 embedding of this graph, but the following is one that was found with the help of MAGma [3]: 


$$
\begin{aligned}
& \rho_{0}=(1,9,6,3,11), \quad \rho_{1}=(2,7,0,10,4), \quad \rho_{2}=(3,8,1,5,11), \quad \rho_{3}=(4,9,2,0,6), \\
& \rho_{4}=(5,1,10,7,3), \quad \rho_{5}=(6,8,11,2,4), \quad \rho_{6}=(7,5,3,0,9), \quad \rho_{7}=(8,6,4,10,1) \text {, } \\
& \rho_{8}=(9,11,5,7,2), \quad \rho_{9}=(10,8,3,6,0), \quad \rho_{10}=(11,9,7,4,1), \quad \rho_{11}=(0,2,5,8,10) \text {. }
\end{aligned}
$$

These rotations give an embedding with 16 faces, of which 6 are triangular, 8 are quadrangular, and 2 have length 5 , giving Euler characteristic $12-30+16=-2$.

- $C_{12}(1,4,6)$

Take $\rho_{i}= \begin{cases}(i+1, i-4, i+4, i+6, i-1) & \text { for } i \text { even } \\ (i+1, i-1, i+6, i+4, i-4) & \text { for } i \text { odd. }\end{cases}$

These rotations give an embedding with 16 faces, of which 4 are triangular and 12 are quadrangular, giving Euler characteristic $12-30+16=-2$.

- $C_{12}(2,3,6)$

As with $C_{12}(1,3,6)$, there seems to be no 'nice' genus 2 embedding of this graph, but the following one was found with the help of MAGMA [3]:

$$
\begin{array}{llll}
\rho_{0}=(2,10,3,9,6), & \rho_{1}=(3,10,11,7,4), & \rho_{2}=(4,8,11,5,0), & \rho_{3}=(5,9,0,1,6), \\
\rho_{4}=(6,1,7,10,2), & \rho_{5}=(7,3,8,2,11), & \rho_{6}=(8,3,4,0,9), & \rho_{7}=(9,5,10,4,1), \\
\rho_{8}=(10,5,6,11,2), & \rho_{9}=(11,6,0,3,7), & \rho_{10}=(0,8,4,7,1), & \rho_{11}=(1,5,2,8,9) .
\end{array}
$$

These rotations give an embedding with 16 faces, of which 6 are triangular, 8 are quadrangular, and 2 have length 5, giving Euler characteristic $12-30+16=-2$.

- $C_{12}(3,4,6)$

Take $\rho_{i}= \begin{cases}(i+3, i+6, i+4, i-4, i-3) & \text { for } i \text { even } \\ (i+3, i-4, i+4, i-3, i+6) & \text { for } i \text { odd. }\end{cases}$

These rotations give an embedding with 16 faces, of which 10 are triangular and 6 have length 5, giving Euler characteristic $12-30+16=-2$.

- $C_{8}(1,2,3,4)$

This is the complete graph $K_{8}$, which by the Ringel-Youngs theorem [10] has minimum genus $\left\lceil\frac{(8-3)(8-4)}{12}\right\rceil=\left\lceil\frac{20}{12}\right\rceil=2$. A genus 2 embedding (with 18 faces) can be found directly by taking $\rho_{i}= \begin{cases}(i+1, i-3, i+2, i-2, i+3, i+4, i-1) & \text { for } i \text { even } \\ (i+1, i-3, i+3, i+4, i-1, i-2, i+2) & \text { for } i \text { odd. }\end{cases}$

- $C_{12}(1,2,4,6)$

Take $\rho_{i}= \begin{cases}(i+1, i+2, i-4, i+4, i+6, i-2, i-1) & \text { for } i \text { even } \\ (i+1, i-1, i-2, i+6, i+4, i-4, i+2) & \text { for } i \text { odd. }\end{cases}$

These give an embedding with 28 triangular faces, and characteristic $12-42+28=-2$.

- $C_{12}(1,4,5,6)$

Take $\rho_{i}= \begin{cases}(i+1, i-5, i-1, i+6, i+5, i+4, i-4) & \text { for } i \text { even } \\ (i+1, i-4, i+4, i+5, i+6, i-1, i-5) & \text { for } i \text { odd. }\end{cases}$

Again these give an embedding with 28 triangular faces, and characteristic -2 . 
The rest of this paper is devoted to showing that the circulants treated above are the only connected circulants of genus 1 or 2 . Recall that every such circulant has valency at most 7 (by Lemma 4), and that all connected circulants with valency $k \leqslant 4$ have minimum genus 0 or 1 . Accordingly, we need only consider valencies 7,5 and 6 , which we do in the next three sections.

\section{Valency 7}

In this section, we assume the valency is 7 , so $n$ must be even, and the circulant has the form $C_{n}\left(a_{1}, a_{2}, a_{3}, \frac{n}{2}\right)$. Moreover, by the final sentence of the proof of Lemma 4 , we have $n \leqslant 12$, and so $n=8,10$ or 12 . We deal with these three cases separately.

Case 7a: $n=8$

Here the only 7 -valent circulant is $C_{8}(1,2,3,4)$, which is isomorphic to $K_{8}$, with minimum genus 2 .

Case 7b: $n=10$

Here there are two possible 7 -valent circulants: $C_{10}(1,2,3,5)$ and $C_{10}(1,2,4,5)$. The first of these is a supergraph of $C_{10}(1,3,5) \cong K_{5,5}$, the genus of which is $\left\lceil\frac{(5-2)^{2}}{4}\right\rceil=3$, and so $\gamma\left(C_{10}(1,2,3,5)\right) \geqslant 3$. The second one is harder to deal with. We explain how it can be shown that $\gamma\left(C_{10}(1,2,4,5)\right)$ is greater than 2 , in Section 7 .

Case 7c: $n=12$

In this case, there are seven non-isomorphic 7 -valent circulants, namely $C_{12}(1,2,3,6)$, $C_{12}(1,2,4,6), C_{12}(1,2,5,6), C_{12}(1,3,4,6), C_{12}(1,3,5,6), C_{12}(1,4,5,6)$ and $C_{12}(2,3,4,6)$.

For each of these, we have $3 F \leqslant 2 E=84$, so a genus 1 embedding is impossible, and a genus 2 embedding requires $F=28$ faces, all of which would have length 3 , giving what we may call a triangular embedding. Both $C_{12}(1,2,4,6)$ and $C_{12}(1,4,5,6)$ have such embeddings; see earlier. In contrast, the circulant $C_{12}(1,3,5,6)$ has genus at least 4 , since it contains $C_{12}(1,3,5) \cong K_{6,6}$ as a subgraph, and $\gamma\left(K_{6,6}\right)=\left\lceil\frac{(6-2)^{2}}{4}\right\rceil=4$.

This leaves $C_{12}(1,2,3,6), C_{12}(1,2,5,6), C_{12}(1,3,4,6)$ and $C_{12}(2,3,4,6)$. Each of these can be shown to have no triangular embedding, and so has minimum genus at least 3 .

For example, take $G=C_{12}(1,3,4,6)$. The only triples of vertices that form a 3 -cycle in $G$ containing vertex 0 are $\{0,1,4\},\{0,1,9\},\{0,3,4\},\{0,3,9\},\{0,3,11\},\{0,4,8\}$, $\{0,6,9\},\{0,8,9\}$ and $\{0,8,11\}$, and so the only possible rotations at vertex 0 for a triangular embedding are $(1,4,8,11,3,6,9)$ and its inverse $(1,9,6,3,11,8,4)$. Then by vertex transitivity, the only possible rotations at each vertex $v$ are $(v+1, v+4, v+8, v+$ $11, v+3, v+6, v+9)$ and its inverse $(v+1, v+9, v+6, v+3, v+11, v+8, v+4)$, each taken $\bmod 12$. But since the rotation at vertex 0 is $(1,4,8,11,3,6,9)$ or $(1,9,6,3,11,8,4)$ and the faces are all triangular, the rotation at vertex 3 must be of the form $(. ., 0,11,6, .$.$) or$ $(. ., 0,6,11, .$.$) , and both of these are impossible. Hence G$ has no triangular embedding.

When $G=C_{12}(2,3,4,6)$, there are no possible rotations at vertex 0 , and when $G=C_{12}(1,2,3,6)$ or $C_{12}(1,2,5,6)$, the existence of a triangular embedding can be ruled out using similar arguments to those for the case of $C_{12}(1,3,4,6)$ above. For $C_{12}(1,2,3,6)$, the only possible rotations at vertex 0 would be $(1,2,3,6,9,10,11),(1,2,3,6,9,11,10)$, 
$(1,2,11,10,9,6,3)$ and $(1,10,9,6,3,2,11)$ and their inverses, but all give no possibility at vertex 3. Similarly, for $C_{12}(1,2,5,6)$, the only rotations at vertex 0 could be $(1,2,7,5,10,11,6)$ and $(1,2,7,6,5,10,11)$ and their inverses, but these give a contradiction at vertices 1 and 5 respectively.

In summary, the only 7 -valent connected circulants that have embeddings of genus 1 or 2 are $C_{8}(1,2,3,4), C_{12}(1,2,4,6)$ and $C_{12}(1,4,5,6)$, all of which have minimum genus 2 .

\section{$5 \quad$ Valency 5}

In this section, we consider circulants of valency 5 , which have the form $C_{n}\left(a_{1}, a_{2}, \frac{n}{2}\right)$, where $n$ is even and $n \geqslant 6$. If such a connected circulant has an embedding of genus 1 or 2 , then the Euler-Poincaré formula gives $-2 \leqslant V-E+F=n-\frac{5 n}{2}+F$, so that $F \geqslant \frac{3 n}{2}-2=\frac{3 n-4}{2}$.

If there are no triangular faces, then $5 n=2 E \geqslant 4 F \geqslant 6 n-8$ and so $n \leqslant 8$. But if $n \leqslant 8$ then up to isomorphism the only possibilities are $C_{6}(1,2,3), C_{8}(1,2,4)$ and $C_{8}(1,3,4)$, and each of these has a genus 1 embedding (by Proposition 5), so we may suppose that at least one face is triangular, and that $n>8$.

For there to be a triangular face, some relation of the form $a_{i} \pm a_{j} \pm a_{k} \equiv 0 \bmod n$ must be satisfied. If $i, j, k$ are distinct, then it is easy to see that $a_{3}=\frac{n}{2}=a_{1}+a_{2}$ in $\mathbb{Z}_{n}$, in which case we have a genus 1 embedding, again by Proposition 5 , so we may assume that at least two of $i, j, k$ coincide. Then since $a_{3}=\frac{n}{2}$, one (or more) of the following must occur: either $2 a_{1} \equiv \pm a_{2} \bmod n$, or $a_{1} \equiv \pm 2 a_{2} \bmod n$, or $\frac{n}{2}=a_{3}=2 a_{1}$ or $2 a_{2}$, or $n=3 a_{1}$ or $3 a_{2}$. Here we will temporarily drop the assumption that $a_{1}<a_{2}$, and then by interchanging $a_{1}$ and $a_{2}$ if necessary, we may suppose that $2 a_{1} \equiv \pm a_{2} \bmod n$, or $\frac{n}{2}=a_{3}=2 a_{2}$, or $n=3 a_{1}$. We will consider these three cases in turn.

Case 5a: $2 a_{1} \equiv \pm a_{2} \bmod n$

Note that connectedness implies that $\operatorname{gcd}\left(a_{1}, 2 a_{1}, \frac{n}{2}, n\right)=1$. If $n \equiv 0 \bmod 4$ then $\frac{n}{2}$ is even so $a_{1}$ is odd, and $\operatorname{gcd}\left(a_{1}, n\right)=1$. Thus $a_{1}$ is invertible $\bmod n$, and we can multiply $X=\left\{a_{1}, a_{2}, a_{3}\right\}=\left\{a_{1}, \pm 2 a_{1}, \frac{n}{2}\right\}$ by its inverse to get $C_{n}\left(a_{1}, a_{2}, a_{3}\right) \cong C_{n}\left(1,2, \frac{n}{2}\right)$, which has genus 1 for $n=8$, and a genus 2 embedding for $n \geqslant 12$. Similarly, if $n \equiv 2 \bmod$ 4, then $\operatorname{gcd}\left(a_{1}, \frac{n}{2}\right)=1$, but in this case there are two possibilities: either $a_{1}$ is odd, and again $C_{n}\left(a_{1}, a_{2}, a_{3}\right) \cong C_{n}\left(1,2, \frac{n}{2}\right)$, which has minimum genus 1 , or $a_{1}$ is even, in which case $\operatorname{gcd}\left(a_{1}, n\right)=2$ and then $C_{n}\left(a_{1}, a_{2}, a_{3}\right) \cong C_{n}\left(2,4, \frac{n}{2}\right)$, which has a genus 2 embedding.

We now prove that $C_{n}\left(1,2, \frac{n}{2}\right)$ has no genus 1 embedding when $n \equiv 0 \bmod 4$ and $n \geqslant 12$, and that $C_{n}\left(2,4, \frac{n}{2}\right)$ has no genus 1 embedding when $n \equiv 2 \bmod 4$ and $n \geqslant 10$.

For $C_{n}\left(1,2, \frac{n}{2}\right)$ with $n \equiv 0 \bmod 4$ and $n \geqslant 12$, we assume the contrary. Then there must be an embedding with $\frac{3 n}{2}$ faces. If $F_{3}$ is the number of triangular faces, and $F_{\ell}$ is the number of faces of length greater than 3 , then counting edge-face incident pairs gives

$$
5 n=2 E \geqslant 3 F_{3}+4 F_{\ell}=4 F-F_{3}=6 n-F_{3},
$$

so that $F_{3} \geqslant n$. Also by counting pairs $(v, \Delta)$ where $\Delta$ is a triangular face at the vertex $v$, we find that the average number of triangular faces at each vertex is at least 
3. But for $C_{n}\left(1,2, \frac{n}{2}\right)$ with $n \geqslant 12$, the only possible triangular faces at the vertex 0 are bounded by vertex-triples $\{0,1,2\},\{0,1,-1\}$ and $\{0,-2,-1\}$, and so the only way to have three or more triangular faces at vertex 0 is to take $\rho_{0}=\left(1,-1,-2, \frac{n}{2}, 2\right)$ or its inverse, both of which give exactly three such faces. It follows that $F_{3}=n$, and so $F_{\ell}=F-F_{3}=\frac{n}{2}$, and all non-triangular faces must have length 4, with two at each vertex. In particular, there must be two faces of length 4 at vertex 0 , and by considering common neighbours of $\frac{n}{2}$ and \pm 2 , it is easy to see that these faces must be bounded by the 4 -cycles $\left(0,2, \frac{n}{2}+2, \frac{n}{2}\right)$ and $\left(0,-2, \frac{n}{2}-2, \frac{n}{2}\right)$. By reflecting the surface if necessary, we can assume $\rho_{0}=\left(1,-1,-2, \frac{n}{2}, 2\right)$. Then $\rho_{1}$ must have the form $(. .,-1,0,2, .$.$) , and so$ $\rho_{1}=\left(2,3, \frac{n}{2}+1,-1,0\right)$, which is the analogue of $\rho_{0}^{-1}$ obtained by adding $1 \bmod n$ to each point of the rotation. By induction, we find that

$$
\rho_{i}= \begin{cases}\left(i+1, i-1, i-2, i+\frac{n}{2}, i+2\right) & \text { for } i \text { even } \\ \left(i+1, i+2, i+\frac{n}{2}, i-2, i-1\right) & \text { for } i \text { odd }\end{cases}
$$

But also the choice of $\rho_{0}$ and the boundaries of the two quadrangular faces at 0 imply that the rotation $\rho_{\frac{n}{2}}$ has the form $\left(. ., \frac{n}{2}+2,0, \frac{n}{2}-2\right)$, and it follows that $\frac{n}{2}$ must be odd. Thus $C_{n}\left(1,2, \frac{n}{2}\right)$ has no genus 1 embedding when $n \equiv 0 \bmod 4$ and $n \geqslant 12$.

For $C_{n}\left(2,4, \frac{n}{2}\right)$ with $n \equiv 2 \bmod 4$ and $n \geqslant 10$, we observe that $C_{n}(2,4)$ is a subgraph, and that this is the union of two copies of $C_{\frac{n}{2}}(1,2)$, on disjoint vertex-sets $\{0,2, \ldots, n-2\}$ and $\{1,3, \ldots, n-1\}$. Then since $\frac{n}{2}$ is odd, we know that $C_{\frac{n}{2}}(1,2)$ has minimum genus 1 , and it follows from the theorems of Battle at al [1] that the minimum genus of $C_{n}\left(2,4, \frac{n}{2}\right)$ is at least 2 (and hence is exactly 2 ).

Case 5b: $\frac{n}{2}=a_{3}=2 a_{2}$

Here $a_{2}=\frac{n}{4}$, and by connectedness, $1=\operatorname{gcd}\left(a_{1}, a_{2}, a_{3}, n\right)=\operatorname{gcd}\left(a_{1}, \frac{n}{4}, \frac{n}{2}, n\right)$. We consider three sub-cases, depending on whether $\operatorname{gcd}\left(a_{1}, n\right)=1,2$ or 4 .

If $\operatorname{gcd}\left(a_{1}, n\right)=1$, then we can multiply $X=\left\{a_{1}, a_{2}, a_{3}\right\}=\left\{a_{1}, \frac{n}{4}, \frac{n}{2}\right\}$ by the inverse of $a_{1} \bmod n$ and find that $C_{n}\left(a_{1}, a_{2}, a_{3}\right) \cong C_{n}\left(1, \frac{n}{4}, \frac{n}{2}\right)$. Now let $F_{3}$ be the number of triangular faces, and let $F_{\ell}$ be the number of faces of length greater than 3 . Then counting edge-face incident pairs and using the inequality $F \geqslant \frac{3 n-4}{2}$ (shown at the beginning of this section) gives $5 n=2 E \geqslant 3 F_{3}+4 F_{\ell}=4 F-F_{3} \geqslant 2(3 n-4)-F_{3}$, so that $n \leqslant F_{3}+8$. But the only possible triangular faces at a vertex $i$ are bounded by vertex-triples of the form $\left\{i, i+\frac{n}{4}, i+\frac{n}{2}\right\},\left\{i, i-\frac{n}{4}, i-\frac{n}{2}\right\}$ or $\left\{i, i+\frac{n}{4}, i-\frac{n}{4}\right\}$, and so there can be at most two at each vertex, and therefore $n-8 \leqslant F_{3} \leqslant \frac{2 n}{3}$, which gives $n \leqslant 24$. Thus $n=8,12$, 16,20 or 24 . We know that the graph $C_{8}(1,2,4)$ has genus 1 , and also that $C_{12}(1,3,6)$ has a genus 2 embedding. On the other hand, $C_{n}\left(1, \frac{n}{4}, \frac{n}{2}\right)$ has no genus 1 embedding for $n \geqslant 12$, since that would require $\frac{3 n}{2}$ faces, with at least $n$ being triangular, so at least three at each vertex. Also we will show in Section 7 that the minimum genus of each of $C_{16}(1,4,8), C_{20}(1,5,10)$ and $C_{24}(1,6,12)$ is greater than 2 .

If $\operatorname{gcd}\left(a_{1}, n\right)=2$, then $a_{1} / 2$ is coprime to $n$ and we can multiply $X=\left\{a_{1}, \frac{n}{4}, \frac{n}{2}\right\}$ by its inverse $\bmod n$ and find that $C_{n}\left(a_{1}, a_{2}, a_{3}\right) \cong C_{n}\left(2, \frac{n}{4}, \frac{n}{2}\right)$. But now $\frac{n}{4}$ must be odd, so the same argument about triangular faces as used in the previous paragraph gives $n=12$ or 20, leaving only $C_{12}(2,3,6)$ and $C_{20}(2,5,10)$ to consider. The first of these has a genus 2 
embedding, but no genus 1 embedding since it can have at most two triangular faces at each vertex, and the second has genus greater than 2, as we explain in Section 7.

Similarly, if $\operatorname{gcd}\left(a_{1}, n\right)=4$, we can multiply $X$ by the inverse of $a_{1} / 4 \bmod n$ and find that $C_{n}\left(a_{1}, a_{2}, a_{3}\right) \cong C_{n}\left(4, \frac{n}{4}, \frac{n}{2}\right)$, with $\frac{n}{4}$ odd, and so $C_{n}\left(a_{1}, a_{2}, a_{3}\right\} \cong C_{12}(4,3,6) \cong$ $C_{12}(3,4,6)$, or $C_{n}\left(a_{1}, a_{2}, a_{3}\right\} \cong C_{20}(4,5,10)$. The graph $C_{12}(3,4,6)$ has a genus 2 embedding but no genus 1 embedding, while the genus of $C_{20}(4,5,10)$ is greater than 2 , as we explain in Section 7.

Case 5c: $n=3 a_{1}$

In this case, $n \equiv 0 \bmod 6$ (since the valency is odd), and $X=\left\{a_{1}, a_{2}, a_{3}\right\}=\left\{\frac{n}{3}, a_{2}, \frac{n}{2}\right\}$. Also we may assume that none of the earlier equations (in 5a or 5b) holds. Under that assumption, the only possible triangular face at each vertex $i$ is bounded by the vertextriple $\left\{i, i+a_{1}, i-a_{1}\right\}$, and so the argument used in case $5 \mathrm{~b}$ about triangular faces gives $n-8 \leqslant F_{3} \leqslant \frac{n}{3}$, which implies that $n \leqslant 12$. Thus $n=6$ or 12 .

When $n=6$ we have $C_{6}(1,2,3)$, which has genus 1 . On the other hand, when $n=12$ we have $a_{1}=4$ and so $a_{2}=1$ or 5 (but not 3 since that possibility was dealt with in $5 \mathrm{~b}$ ). This leaves $C_{12}(4,1,6)$ and $C_{12}(4,5,6)$, both of which are isomorphic to $C_{12}(1,4,6)$. The latter has a genus 2 embedding, but no genus 1 embedding, since a genus 1 embedding would require 18 faces, with at least 12 being triangular, but the graph contains only four simple 3 -cycles (viz. those bounded by $\{0,4,8\},\{1,5,9\},\{2,6,10\}$ and $\{3,7,11\}$ ).

\section{Valency 6}

In this section we consider connected circulants $C_{n}\left(a_{1}, a_{2}, a_{3}\right)$ with $0<a_{1}<a_{2}<a_{3}<\frac{n}{2}$. By the Euler-Poincaré formula, the number of faces in an embedding of genus 1 or 2 must be $2 n$ or $2 n-2$, respectively. Also if we let $F_{3}$ be the number of triangular faces and $F_{\ell}$ the number of faces of length greater than 3 , then counting edge-face incident pairs gives $6 n=2 E \geqslant 3 F_{3}+4 F_{\ell}=4 F-F_{3}=8 n-F_{3}$ or $8 n-8-F_{3}$, so that $F_{3} \geqslant 2 n$ or $2 n-8$ respectively. Moreover, if the average number of triangular faces at a vertex is at most 3 , then $2 n-8 \leqslant F_{3} \leqslant n$, so $n \leqslant 8$, and the only possibilities would be $C_{7}(1,2,3)$ and $C_{8}(1,2,3)$, both of which have genus 1 (by Proposition 5). Hence we may suppose there are at least four triangular faces at some vertex, and by vertex-transitivity, we might as well assume that this happens at vertex 0 .

As earlier, we observe that having a triangular face implies some relation of the form $a_{i} \pm a_{j} \pm a_{k} \equiv 0 \bmod n$. Moreover, if $i, j, k$ are distinct, then by the proof of Proposition 5, there exists a genus 1 embedding, covered by the second item of Theorem 2 .

So we will assume that no such relation holds with $i, j, k$ are distinct. Then there are only 12 other possibilities, in three categories as follows:

(T1) $a_{2}=2 a_{1}$, or (T2) $a_{3}=2 a_{1}$, or (T3) $a_{3}=2 a_{2}$;

(T4) $2 a_{1}+a_{2}=n$, or (T5) $2 a_{1}+a_{3}=n$, or (T6) $a_{1}+2 a_{2}=n$,

or (T7) $2 a_{2}+a_{3}=n$, or (T8) $a_{1}+2 a_{3}=n$, or (T9) $a_{2}+2 a_{3}=n$;

(T10) $3 a_{1}=n$, or (T11) $3 a_{2}=n$, or (T12) $3 a_{3}=n$. 
Each such relation gives rise to at most three triangular faces at a vertex, so to get an embedding of genus 1 or 2 (not already covered by the second item of Theorem 2), we need to assume that at least two of these relations hold.

Moreover, those relations must involve all three of $a_{1}, a_{2}$ and $a_{3}$, for if they involve only $a_{i}$ and $a_{j}$, say, then only possible triangular faces at vertex 0 are bounded by triples made up of 0 and two of $\pm a_{i}$ and $\pm a_{j}$, and no more than three of these can be taken together in a candidate for the rotation $\rho_{0}$. Also some of the combinations of these relations (involving all three of $a_{1}, a_{2}$ and $a_{3}$ ) are inconsistent.

It is an easy exercise to show that there are just 20 combinations that are feasible, namely the 14 pairs $\{\mathrm{T} i, \mathrm{~T} j\}$ for $\{i, j\}=\{1,3\},\{1,7\},\{1,8\},\{1,9\},\{1,12\},\{2,6\},\{2,7\}$, $\{2,9\},\{2,11\},\{3,8\},\{5,6\},\{5,11\},\{7,8\}$ and $\{8,11\}$, and the six triples $\{\mathrm{T} i, \mathrm{~T} j, \mathrm{~T} k\}$ for $\{i, j, k\}=\{1,3,8\},\{1,3,9\},\{1,3,12\},\{1,8,11\},\{2,7,8\}$ and $\{2,8,11\}$. We deal with these one-by-one below.

Case 6a: $\{\mathrm{T} 1, \mathrm{~T} 3\}$, with $a_{2}=2 a_{1}$ and $a_{3}=2 a_{2}$

Here $X=\left\{a_{1}, 2 a_{1}, 4 a_{1}\right\}$, so $\operatorname{gcd}\left(a_{1}, n\right)=1$, and then $C_{n}\left(a_{1}, 2 a_{1}, 4 a_{1}\right) \cong C_{n}(1,2,4)$. For the latter graph, the only possibilities for a triangular face at vertex 0 are bounded by the triples $\{0,1,2\},\{0,2,4\},\{0,1,-1\},\{0,-1,-2\},\{0,-2,-4\}$ and $\{0,-2,2\}$.

For $n>12$, there is no way to arrange these to give six triangular faces at vertex 0 , and so there cannot be six triangular faces at any vertex. Moreover, the only way to get five triangular faces at vertex 0 is to take $\rho_{0}$ as either $(4,2,1,-1,-2,-4)$ or its inverse, but in that case $\rho_{2}$ must be of the form $(. ., 1,0,4, .$.$) or (. ., 4,0,1, .$.$) , so \rho_{2}$ cannot be $(6,4,3,1,0,-2)$ or its inverse. It follows that if there are five five triangular faces at a vertex $v$, then there are at most four triangular faces at vertex $v+2$. Hence if $r$ is the number of vertices that lie in five triangular faces, and $s$ is the number that do not, we have $r \leqslant s$, and so $r \leqslant \frac{n}{2}$. Now using the inequality $F_{3} \geqslant 2 n-8$ (shown at the beginning of this section) and counting pairs $(v, \Delta)$ where $\Delta$ is a triangular face at the vertex $v$, we have $3(2 n-8) \leqslant 3 F_{3} \leqslant 5 r+4 s=4(r+s)+r=4 n+r \leqslant 4 n+\frac{n}{2}$, and therefore $n \leqslant 16$. Hence we need only consider $C_{n}(1,2,4)$ for $8 \leqslant n \leqslant 16$.

The graphs $C_{8}(1,2,4)$ and $C_{9}(1,2,4)$ both have genus 1 , and each of $C_{10}(1,2,4)$ and $C_{12}(1,2,4)$ has a genus 2 embedding, by Proposition 6 .

On the other hand, $C_{10}(1,2,4)$ has no genus 1 embedding, because that would require 20 faces, all triangular, and an examination of the possibilities for triangular faces at vertex 0 shows that the rotation $\rho_{0}$ would have to be $(1,2,4,6,9,8)$ or its inverse, or $(1,2,6,4,9,8)$ or its inverse. The first of these (and its inverse) makes it impossible to find a suitable $\rho_{2}$, so we may suppose that $\rho_{0}=(1,2,6,4,9,8)$, but then $\rho_{1}$ and $\rho_{2}$ are both analogues of $\rho_{0}^{-1}$, and so this quickly leads to a contradiction.

Similarly, a genus 1 embedding of $C_{12}(1,2,4)$ would require 24 triangular faces, with six at each vertex. This forces $\rho_{0}$ to be $(1,2,4,8,10,11)$ or its inverse, and analogously, $\rho_{2}$ must be $(3,4,6,10,0,1)$ or its inverse, but that is impossible since the ordering of triangular faces at vertex 0 forces $\rho_{2}$ to have the form $(. ., 4,0,1, .$.$) or (. ., 1,0,4, .$.$) .$

Finally, for $n \in\{11,13,14,15,16\}$ a genus 1 embedding would require $2 n$ faces, all triangular, but that is impossible since there can be only one triangular face containing the edge $\{0,4\}$, namely one that has 2 as its third vertex. In Section 7 we explain why 
none of these five graphs can have an embedding of genus 2 .

Case 6b: $\{\mathrm{T} 1, \mathrm{~T} 7\}$, with $a_{2}=2 a_{1}$ and $2 a_{2}+a_{3}=n$ 6 a.

Here $C_{n}\left(a_{1}, a_{2}, a_{3}\right)=C_{n}\left(a_{1}, 2 a_{1}, n-4 a_{1}\right) \cong C_{n}\left(a_{1}, 2 a_{1}, 4 a_{1}\right)$, which was met in case

Case 6c: $\quad\{\mathrm{T} 1, \mathrm{~T} 8\}$, with $a_{2}=2 a_{1}$ and $a_{1}+2 a_{3}=n$

In this case, we have $a_{3}=\frac{n-a_{1}}{2}$, and also $1=\operatorname{gcd}\left(a_{1}, 2 a_{1}, \frac{n-a_{1}}{2}, n\right)=\operatorname{gcd}\left(a_{1}, \frac{n-a_{1}}{2}, n\right)$. Let $d=\operatorname{gcd}\left(a_{1}, n\right)$. Then $1=\operatorname{gcd}\left(a_{1}, \frac{n-a_{1}}{2}, n\right)=d$ or $\frac{d}{2}$, so $d=1$ or 2 .

If $d=1$ then $a_{1}$ is a unit $\bmod n$, and since $n-a_{1}=2 a_{3}$ is even, both $a_{1}$ and $n$ are odd, and therefore we can multiply by twice the inverse $b$ of $a_{1} \bmod n$ and find that $C_{n}\left(a_{1}, a_{2}, a_{3}\right) \cong C_{n}\left(2,4, b\left(n-a_{1}\right)\right) \cong C_{n}(2,4,-1) \cong C_{n}(1,2,4)$, as in case 6 a.

On the other hand, if $d=2$, then both $a_{1}$ and $n$ are even, but $a_{3}=\frac{n-a_{1}}{2}$ is odd, and so $\frac{n+a_{1}}{2}=\frac{n-a_{1}}{2}+a_{1}$ is odd, and therefore $\operatorname{gcd}\left(n, \frac{n+a_{1}}{2}\right)=\operatorname{gcd}\left(\frac{n}{2}, \frac{n+a_{1}}{2}\right)=\operatorname{gcd}\left(\frac{n}{2}, \frac{a_{1}}{2}\right)=1$. Letting $x$ be $\frac{n+a_{1}}{2}$, and $y$ be its inverse $\bmod n$, we have $2 x=n+a_{1} \equiv a_{1} \bmod n$ and $a_{3}=x-a_{1}$, so multiplying by $y$ we have $2 \equiv y a_{1} \bmod n$ and $y a_{3} \equiv 1-y a_{1} \bmod n$, and therefore $C_{n}\left(a_{1}, a_{2}, a_{3}\right) \cong C_{n}\left(y a_{1}, 2 y a_{1}, y a_{3}\right) \cong C_{n}(2,4,-1) \cong C_{n}(1,2,4)$, again as in 6a.

Case 6d: $\{\mathrm{T} 1, \mathrm{~T} 9\}$, with $a_{2}=2 a_{1}$ and $a_{2}+2 a_{3}=n$

Here $n$ is even and $a_{1}+a_{3}=\frac{n}{2}$, with $a_{2}=2 a_{1} \equiv-2 a_{3} \bmod n$, and it follows that $\operatorname{gcd}\left(a_{1}, n\right)=1$ or $\operatorname{gcd}\left(a_{3}, n\right)=1$. If $\operatorname{gcd}\left(a_{1}, n\right)=1$, then $a_{1}$ is odd, and we can multiply by its inverse $\bmod n$ and find that $C_{n}\left(a_{1}, a_{2}, a_{3}\right) \cong C_{n}\left(1,2, \frac{n}{2}-1\right)$. On the other hand, if $\operatorname{gcd}\left(a_{3}, n\right)=1$, then $a_{3}$ is odd and we can multiply by its inverse $\bmod n$ to show that $C_{n}\left(a_{1}, a_{2}, a_{3}\right) \cong C_{n}\left(a_{3}, a_{2}, a_{1}\right) \cong C_{n}\left(1,-2, \frac{n}{2}-1\right) \cong C_{n}\left(1,2, \frac{n}{2}-1\right)$.

If $n \equiv 0 \bmod 4$, this circulant has genus 1 , by Proposition 5, and if $n \equiv 2 \bmod 4$, then it has a genus 2 embedding, by Proposition 6 . Also the graph $C_{10}(1,2,4)$ was shown to have genus 2 in case 6 a.

We now prove that $C_{n}\left(1,2, \frac{n}{2}-1\right)$ has no genus 1 embedding when $n \equiv 2 \bmod 4$ and $n>10$. To do this, we assume the contrary. Then $C_{n}\left(1,2, \frac{n}{2}-1\right)$ has an embedding with $2 n$ faces, all of which must be triangular. The only possibilities for a triangular face at vertex 0 are bounded by the triples $\{0,1,2\},\{0,1,-1\},\left\{0,2, \frac{n}{2}+1\right\},\left\{0, \frac{n}{2}-1, \frac{n}{2}+1\right\}$, $\left\{0, \frac{n}{2}-1,-2\right\}$ and $\{0,-2,-1\}$, so the only possibilities for the rotation $\rho_{0}$ are $\left(1,2, \frac{n}{2}+\right.$ $\left.1, \frac{n}{2}-1,-2,-1\right)$ and its inverse. By reflecting the surface if necessary, we can assume that $\rho_{0}=\left(1,2, \frac{n}{2}+1, \frac{n}{2}-1,-2,-1\right)$. Then $\rho_{1}$ must be of the form $(. ., 2,0,-1, .$.$) , and it$ follows that $\rho_{1}=\left(2,0,-1, \frac{n}{2}, \frac{n}{2}+2,3\right)$, which is the analogue of $\rho_{0}^{-1}$. Similarly, $\rho_{2}$ must be of the form $\left(. ., \frac{n}{2}+1,0,1, ..\right)$, so $\rho_{2}=\left(3,4, \frac{n}{2}+3, \frac{n}{2}+1,0,1\right)$, which is the analogue of $\rho_{0}$. By induction, $\rho_{i}$ is equal to the analogue of $\rho_{0}$ when $i$ is even, and of $\rho_{0}^{-1}$ when $i$ is odd (as given for the genus 1 embedding of $C_{n}\left(1,2, \frac{n}{2}-1\right)$ when $n \equiv 0 \bmod 4$ in the proof of Proposition 5). But then since $\frac{n}{2}-1$ is even, $\rho_{\frac{n}{2}-1}$ should be $\left(\frac{n}{2}, \frac{n}{2}+1,0,-2, \frac{n}{2}-3, \frac{n}{2}-1\right)$, which is not the case, since consideration of the faces containing the edge $\left\{0, \frac{n}{2}-1\right\}$ shows that $\rho_{\frac{n}{2}-1}$ must have the form $\left(. .,-2,0, \frac{n}{2}+1, ..\right)$.

Thus $C_{n}\left(1,2, \frac{n}{2}-1\right)$ has genus 2 when $n \equiv 2 \bmod 4$ and $n \geqslant 10$.

Case 6e: $\{\mathrm{T} 1, \mathrm{~T} 12\}$, with $a_{2}=2 a_{1}$ and $3 a_{3}=n$

In this case, at vertex 0 we can get at most three triangular faces from the relation $a_{2}=2 a_{1}$ and at most one from the relation $3 a_{3}=n$ (namely a face bounded by the triple 
$\left.\left\{0, a_{3},-a_{3}\right\}=\left\{0, \frac{n}{3}, \frac{2 n}{3}\right\}\right)$, so at most four triangular faces at any vertex. It follows that $2 n-8 \leqslant F_{3} \leqslant \frac{4 n}{3}$, and therefore $n \leqslant 12$. Then since $n=3 a_{3}$ is divisible by 3 , we have only $C_{9}(1,2,3)$, which has genus 1 , and $C_{12}(1,2,4)$, which was met in case 6 a.

Case 6f: $\quad\{\mathrm{T} 2, \mathrm{~T} 6\}$, with $a_{3}=2 a_{1}$ and $a_{1}+2 a_{2}=n$

This case is analogous to $6 \mathrm{c}$, with the roles of $a_{2}$ and $a_{3}$ interchanged. Here we have $C_{n}\left(a_{1}, a_{2}, a_{3}\right) \cong C_{n}\left(a_{1}, \frac{n-a_{1}}{2}, 2 a_{1}\right) \cong C_{n}\left(a_{1}, 2 a_{1}, \frac{n-a_{1}}{2}\right) \cong C_{n}(1,2,4)$, which was met in 6 a.

Case 6g: $\{\mathrm{T} 2, \mathrm{~T} 7\}$, with $a_{3}=2 a_{1}$ and $2 a_{2}+a_{3}=n$

This case is analogous to $6 \mathrm{~d}$, with the roles of $a_{2}$ and $a_{3}$ interchanged. Here we have $X=\left\{a_{1}, a_{2}, a_{3}\right\}=\left\{a_{1}, \frac{n}{2}-a_{1}, 2 a_{1}\right\}$, so $\operatorname{gcd}\left(a_{1}, n\right)=1$, and then multiplying by the inverse of $a_{1} \bmod n$ we find that $C_{n}\left(a_{1}, a_{2}, a_{3}\right) \cong C_{n}\left(1, \frac{n}{2}-1,2\right) \cong C_{n}\left(1,2, \frac{n}{2}-1\right)$, which was met in case $6 \mathrm{~d}$.

Case 6h: $\{$ T2, T9 $\}$, with $a_{3}=2 a_{1}$ and $a_{2}+2 a_{3}=n$

Here we have $X=\left\{a_{1}, a_{2}, a_{3}\right\}=\left\{a_{1}, n-4 a_{1}, 2 a_{1}\right\}$, and so $\operatorname{gcd}\left(a_{1}, n\right)=1$. Multiplying by the inverse of $a_{1} \bmod n$ we find $C_{n}\left(a_{1}, a_{2}, a_{3}\right) \cong C_{n}(1,-4,2) \cong C_{n}(1,2,4)$, met in $6 \mathrm{a}$.

Case 6i: $\quad\{\mathrm{T} 2, \mathrm{~T} 11\}$, with $a_{3}=2 a_{1}$ and $3 a_{2}=n$

This case is analogous to $6 \mathrm{e}$, with the roles of $a_{2}$ and $a_{3}$ interchanged. In particular, we have at most four triangular faces at a vertex, so $n \leqslant 12$, giving $n=9$ or 12 . If $n=9$ then we have $C_{9}(2,3,4) \cong C_{9}(1,6,2) \cong C_{9}(1,2,3)$, which has genus 1 , while there is no possibility when $n=12$.

Case 6j: $\{$ T3, T8 $\}$, with $a_{3}=2 a_{2}$ and $a_{1}+2 a_{3}=n$

This case is analogous to $6 \mathrm{~h}$, with the roles of $a_{1}$ and $a_{2}$ interchanged, and so gives only the graph $C_{n}(1,2,4)$, met in case 6 a.

Case 6k: $\{$ T5, T6 $\}$, with $2 a_{1}+a_{3}=n$ and $a_{1}+2 a_{2}=n$

Here $C_{n}\left(a_{1}, a_{2}, a_{3}\right) \cong C_{n}\left(-2 a_{2}, a_{2}, 4 a_{2}\right)$, and then since $\operatorname{gcd}\left(a_{2}, n\right)=1$, we can multiply by the inverse of $a_{2} \bmod n$ and find $C_{n}\left(a_{1}, a_{2}, a_{3}\right) \cong C_{n}(-2,1,4) \cong C_{n}(1,2,4)$, met in 6 a.

Case 61: $\{\mathrm{T} 5, \mathrm{~T} 11\}$, with $2 a_{1}+a_{3}=n$ and $3 a_{2}=n$

This is similar to cases $6 \mathrm{e}$ and $6 \mathrm{i}$. At vertex 0 we can get at most three triangular faces from the relation $2 a_{1}+a_{3}=n$ and at most one from the relation $3 a_{2}=n$, so at most four triangular faces at any vertex. Again it follows that $n=9$ or 12 , but this time there are no possibilities for either value of $n$.

Case 6m: $\{\mathrm{T} 7, \mathrm{~T} 8\}$, with $2 a_{2}+a_{3}=n$ and $a_{1}+2 a_{3}=n$

Here $C_{n}\left(a_{1}, a_{2}, a_{3}\right) \cong C_{n}\left(4 a_{2}, a_{2},-2 a_{2}\right)$, and then since $\operatorname{gcd}\left(a_{2}, n\right)=1$, we can multiply by the inverse of $a_{2} \bmod n$ and find $C_{n}\left(a_{1}, a_{2}, a_{3}\right) \cong C_{n}(4,1,-2) \cong C_{n}(1,2,4)$, met in 6 a.

Case 6n: $\{$ T8, T11 $\}$, with $a_{1}+2 a_{3}=n$ and $3 a_{2}=n$

This case is analogous to $6 \mathrm{l}$, with the roles of $a_{1}$ and $a_{3}$ interchanged. Again we have $n=9$ or 12 , and this time there are two possibilities: $C_{9}(1,3,4)$, which has genus 1 , and $C_{12}(2,4,5) \cong C_{12}(5,-2,-4) \cong C_{12}(1,2,4)$, met in 6 a.

Cases 6o to 6s: $\{\mathrm{T} 1, \mathrm{~T} 3, \mathrm{~T} 8\},\{\mathrm{T} 1, \mathrm{~T} 3, \mathrm{~T} 9\},\{\mathrm{T} 1, \mathrm{~T} 3, \mathrm{~T} 12\},\{\mathrm{T} 1, \mathrm{~T} 8, \mathrm{~T} 11\}\{\mathrm{T} 2, \mathrm{~T} 7, \mathrm{~T} 8\}$

In each of these cases, the three relations and connectedness give a unique graph that has been considered already. For example, in case $6 \mathrm{~s}$ (for the triple $\{\mathrm{T} 2, \mathrm{~T} 7, \mathrm{~T} 8\}$ ) we have $a_{3}=2 a_{1}$ and $2 a_{2}+a_{3}=n$ and $a_{1}+2 a_{3}=n$, which give $n-a_{1}=2 a_{3}=4 a_{1}$, and therefore 
$\left(a_{1}, a_{2}, a_{3}\right)=\left(\frac{n}{5}, \frac{3 n}{10}, \frac{2 n}{5}\right)$; then by connectedness $1=\operatorname{gcd}\left(n, \frac{n}{5}, \frac{3 n}{10}, \frac{2 n}{5}\right)=\frac{n}{10}$, so $n=10$, and we have $C_{10}(2,3,4) \cong C_{10}(1,2,4)$, which was met in 6 a. Similarly, the cases 6 o to $6 \mathrm{r}$ give $C_{9}(1,2,4), C_{10}(1,2,4), C_{12}(1,2,4)$, and $C_{12}(2,4,5) \cong C_{12}(1,2,4)$.

Case 6t: $\quad\{\mathrm{T} 2, \mathrm{~T} 8, \mathrm{~T} 11\}$, with $a_{3}=2 a_{1}$ and $a_{1}+2 a_{3}=n$ and $3 a_{2}=n$

In this final case, again $n-a_{1}=2 a_{3}=4 a_{1}$, and this time $\left(a_{1}, a_{2}, a_{3}\right)=\left(\frac{n}{5}, \frac{n}{3}, \frac{2 n}{5}\right)$, so by connectedness $1=\operatorname{gcd}\left(n, \frac{n}{5}, \frac{n}{3}, \frac{2 n}{5}\right)=\frac{n}{15}$, which gives $n=15$ and we have $C_{15}(3,5,6)$. But for this graph there can be at most four triangular faces at any vertex, so at most 20 triangular faces in total, and an embedding of genus 1 or 2 is therefore impossible.

\section{$7 \quad$ Remaining cases}

In this section we deal with the remaining unresolved cases, some of which are rather more difficult than others.

To complete the proof of Theorems 2 and 3, we still need to show or explain why $C_{12}(3,4,6)$ has no genus 1 embedding, and why the minimum genus of each of the circulants $C_{n}(1,2,4)$ for $n \in\{11,13,14,15,16\}$ and $C_{16}(1,4,8), C_{20}(1,5,10), C_{20}(2,5,10)$, $C_{20}(4,5,10), C_{24}(1,6,12)$ and $C_{10}(1,2,4,5)$ is greater than 2 . We treat these cases below, but in a slightly different order, still beginning with a case from Theorem 3 .

- $C_{12}(3,4,6)$ has no genus 1 embedding

In this case, a genus 1 embedding needs 18 faces, at least 12 of which are triangular, so the average number of triangular faces at each vertex is at least 3 . On the other hand, there are only four vertex-triples that can bound a triangular face at vertex 0 , namely $\{0,3,6\},\{0,3,9\},\{0,6,9\}$ and $\{0,4,8\}$, and at most three of these can be taken together. Hence there must be 12 triangular faces, with three at each vertex. Moreover, two of the three triangular faces at a given vertex share a common edge, and in each case, the vertices of those two triangles must all differ by multiples of 3 , while the other triangular face is bounded by one of the triples $\{0,4,8\},\{1,5,9\},\{2,6,10\}$ and $\{3,5,11\}$.

Counting edge-face incident pairs gives the average face-size of the larger faces as 4 , and so the six non-triangular faces must be quadrangular. The rotation at vertex 0 can be taken as $(4,8, x, y, z)$, where $(x, y, z)$ is some permutation of $(3,6,9)$, and then the two quadrangular faces at 0 are bounded by 4 -cycles of the form $(x, 0,8, u)$ and $(4,0, z, v)$. Also by considering common neighbours of 4 and $z$, we see that $(z, v)=(3,7),(6,10)$ or $(9,1)$, and note that in each case the difference $z-v$ is \pm 4 .

Now we consider what happens at the vertex $y$. The edge $\{0, y\}$ lies in two triangular faces bounded by the triples $\{0, x, y\}$ and $\{0, y, z\}$, so the other face containing the edge $\{y, z\}$ must be quadrangular, bounded say by the 4 -cycle $(z, y, p, q)$. Then at vertex $z$, the triangular face bounded by the triple $\{0, y, z\}$ sits in between the two quadrangular faces bounded by the 4-cycles $(4,0, z, v)$ and $(z, y, p, q)$. It follows that the other two faces at vertex $z$ must be triangular, and adjacent, but that is impossible, since the difference $v-z$ is not divisible by 3 . Hence no genus 1 embedding exists, and so $C_{12}(3,4,6)$ has genus 2 . 
Next, recall that we showed in case $5 \mathrm{~b}$ of Section 5 that $C_{n}\left(1, \frac{n}{4}, \frac{n}{2}\right)$ has no genus 1 embedding for $n \geqslant 12$, but has one of genus 2 for $n=12$, and we claimed that it has no genus 2 embedding for $n=16,20$ or 24 . We verify that claim now.

- $C_{n}\left(1, \frac{n}{4}, \frac{n}{2}\right)$ has no embedding of genus 2 , for $n=16,20$ or 24

In each case, a genus 2 embedding requires $\frac{3 n-4}{2}$ faces, but at any vertex there are at most two triangular faces, so if $F_{3}$ is the number of triangular faces, then $n-8 \leqslant F_{3} \leqslant \frac{2 n}{3}$. Moreover, if there are two triangular faces at the vertex 0 , then the rotation $\rho_{0}$ must be of the form $(x, y, z, 1,-1)$ or $(x, y, z,-1,1)$, where $\{x, y, z\}=\left\{\frac{n}{4}, \frac{n}{2},-\frac{n}{4}\right\}$, and then since 0 is the only common neighbour of 1 and -1 , the face at 0 containing the edges $\{0,1\}$ and $\{0,-1\}$ must have length at least 5 .

Now if $n=24$, then the conditions $n-8 \leqslant F_{3} \leqslant \frac{2 n}{3}$ force $F_{3}$ to be 16 , and also there must be exactly two triangular faces at each vertex, and therefore at least one face of length 5 or more. But on the other hand, the remaining $\frac{72-4}{2}-16=18$ faces use at most $2 E-3 F_{3}=120-48=72$ edges, so they must all be quadrangular, contradiction.

Similarly, if $n=20$, then there are $\frac{60-4}{2}=28$ faces, with 12 or 13 being triangular, and so there must two triangular faces at 16 or more vertices. If $F_{3}=12$, then the 16 non-triangular faces use at most $2 E-3 F_{3}=100-36=64$ edges, so they must all be quadrangular, which is impossible. Hence $F_{3}=13$, and the 15 non-triangular faces use at most $100-39=61$ edges, so there must be 14 quadrangular faces, plus one of length 5. This, however, implies that there can be at most five vertices that are incident with two triangular faces, again impossible.

Finally, suppose $n=16$. Then there are 22 faces, with 8,9 or 10 of these being triangular, and the number of vertices incident with two triangular faces is at least 8,11 or 14 respectively. If $F_{3}=8$ then the other 14 faces use at most $2 E-3 F_{3}=80-24=56$ edges, so they must all be quadrangular, contradiction. Similarly, if $F_{3}=9$ then the other 13 faces use at most $80-27=53$ edges, so there must be 12 quadrangular faces, plus one of length 5 , and hence there can be at most five vertices that are incident with two triangular faces, again impossible. Thus $F_{3}=10$. But now the other 12 faces use at most $80-30=50$ edges, so there must be either 10 quadrangular faces and two of length 5 , or 11 quadrangular faces and one of length 6 , and then the number of of vertices incident with two triangular faces is at most $2 \cdot 5=10$ or $1 \cdot 6=6$ (respectively), so we reach a contradiction in both cases.

The same kind of argument works for the next two 5-valent circulants as well.

- $C_{20}(2,5,10)$ and $C_{20}(4,5,10)$ have no embedding of genus 1 or 2

These cases are similar to $C_{20}(1,5,10)$. The only possibilities for a triangular face at vertex 0 are bounded by the triples $\{0,5,10\},\{0,5,15\}$ and $\{0,10,15\}$, and so in any embedding, there can be at most two triangular faces at a vertex, and at most 13 triangular faces in total. Moreover, if there are two triangular faces at vertex 0 , then the other two edges (viz. $\{0,2\}$ and $\{0,-2\}$, or $\{0,4\}$ and $\{0,-4\}$ ) lie in a face of length greater than 4.

A genus 1 embedding would require 30 faces, with at least 20 being triangular, which is impossible. On the other hand, a genus 2 embedding requires 28 faces, and just as in 
the case of $C_{20}(1,5,10)$ above, needs 12 or 13 of these to be triangular, with two triangular faces occurring at 16 or more vertices; but again there are either 12 triangular and 16 quadrangular faces, or 13 triangular and 14 quadrangular faces and one of length 5, and in both cases we obtain a contradiction.

Next, we deal with the only remaining 7 -valent case.

- $C_{10}(1,2,4,5)$ has no embedding of genus 1 or 2

A genus 1 embedding would require 25 faces, which is impossible since these faces would use at least $\frac{25 \cdot 3}{2}=\frac{75}{2}$ edges, which is too many. (Also $C_{10}(1,2,4,5)$ has $C_{10}(1,2,4)$ as a subgraph, and we know the genus of $C_{10}(1,2,4)$ is 2.)

Now let us suppose $C_{10}(1,2,4,5)$ has an embedding of genus 2 . This must have 23 faces, of which 22 are triangular and one has length 4 . In contrast to earlier situations, there are lots of possibilities (in fact a total of 66) for the rotation at a given vertex that can make all faces at that vertex triangular, so instead, we can focus on the single face of length 4. By vertex-transitivity, we may suppose this face contains vertex 0. Even then, there are 160 possibilities for the 4-cycle bounding the face, but up to reflection and automorphisms of $C_{10}(1,2,4,5)$ this reduces to 41 possibilities. We were not able to find a short argument to eliminate all of them, and resorted to using a computer (and specifically, the MAGMA system [3]) to help prove there is no genus 2 embedding.

For each possibility for the 4-cycle $(p, q, r, s)$ bounding the sole quadrangular face, we know that the side-edges $\{p, q\},\{q, r\},\{r, s\}$ and $\{s, p\}$ lie also in triangular faces bounded by triples $\{p, t, q\},\{q, u, r\},\{r, v, s\}$ and $\{s, w, p\}$, and the numbers of possibilities for $t, u, v$ and $w$ are limited. Moreover, for any choice of $u, v, w$ and $x$ we know four consecutive points of the rotations at each of the vertices $p, q, r$ and $s$; for example, $\rho_{q}$ must be of the form $(. ., u, r, p, t, .$.$) . This reduces the number of possibilities$ for those rotations to at most $3 !=6$, and often fewer, and sometimes to zero. For example, if $(p, q, r, s)=(0,1,3,8)$ and $(t, u, v)=(2,5,7)$ (and $w=4,6$ or 9$)$, then $\rho_{p}=(2,1,8,4,9,5,6), \rho_{q}=(5,3,0,2,6,7,9)$ and $\rho_{r}=(7,8,1,5,9,4,2)$, but that forces $\rho_{2}$ to have the form $(. ., 6,1,0,6, .$.$) ; contradiction.$

Our computation took less than an hour using MAgma on a laptop, and showed that all other possibilities either lead to a similar contradiction, or force choices for rotations at other vertices that in turn are impossible. (It also found that many possibilities provide an embedding of genus 3 , so the minimum genus of $C_{10}(1,2,4,5)$ is 3 .)

This brings us to the most challenging family, namely the circulants $C_{n}(1,2,4)$ for $n \in\{11,13,14,15,16\}$. We know these have no embedding of genus 1 , since we cannot have all faces triangular. In particular, there are at most five triangular faces at any vertex. Also a genus 2 embedding requires $2 n-2$ faces, with at least $2 n-8$ being triangular.

Now let $V_{5}$ be the number of vertices incident with 5 triangular faces. Then there are at most 4 triangular faces at each of the other $n-V_{5}$ vertices, so counting pairs $(v, \Delta)$ pairs as previously gives $V_{5}+4 n=5 V_{5}+4\left(n-V_{5}\right) \geqslant 3 F_{3} \geqslant 3(2 n-8)=6 n-24$, and therefore $V_{5} \geqslant 2 n-24$. In particular, for $n \in\{13,14,15,16\}$, at least one vertex must be incident with 5 triangular faces. 
Next, suppose that the vertex 0 is incident with 5 triangular faces. Then since the only triangular faces containing the edges $\{0,4\}$ and $\{0,-4\}$ are bounded by the triples $\{0,2,4\}$ and $\{0,-2,-4\}$, the rotation $\rho_{0}$ must be $(-4,-2,-1,1,2,4)$ or its inverse. By reflecting the surface if necessary, we may suppose that $\rho_{0}=(-4,-2,-1,1,2,4)$. Then $\rho_{2}$ must have the form $(. ., 4,0,1, .$.$) , which is not analogous to \rho_{0}$ or $\rho_{0}^{-1}$, and it follows that the vertex 2 cannot be incident with 5 triangular faces. Similarly, if the vertex 3 were incident with 5 triangular faces, then $\rho_{3}$ would have to be $(-1,1,2,4,5,7)$ or its inverse, but then $\rho_{2}$ would have to be of the form $(. ., 4,3,1, .$.$) or (. ., 1,3,4 .$.$) , both of which are$ impossible since $\rho_{2}$ has the form $(4,0,1, p, q, r)$ for some $p, q, r$.

Also if vertices 0 and 1 are both incident with 5 triangular faces, then it is impossible for both of vertices 5 and 6 to be, for otherwise if (say) $\rho_{0}=(-4,-2,-1,1,2,4)$, then we would have $\rho_{1}=(5,3,2,0,-1,-3)$ and then $\rho_{5}=(9,7,6,4,3,1)$ and then $\rho_{6}=$ $(2,4,5,7,8,10)$, but in that case the edge $\{2,4\}$ is used in triangular faces with the same orientation at vertices 0 and 6 ; contradiction.

By vertex-transitivity, it follows that if a vertex $v$ is incident with 5 triangular faces, then $v \pm 2$ and $v \pm 3$ are not. We can now proceed, by considering possibilities for the sequence $Y=\left(y_{0}, y_{1}, y_{2}, \ldots, y_{n-1}\right)$, where

$$
y_{i}= \begin{cases}1 & \text { if vertex } i \text { is incident with } 5 \text { triangular faces, or } \\ 0 & \text { if not. }\end{cases}
$$

At least $2 n-24$ terms of this sequence must be 1 , but also these two conditions must hold:

(a) if $y_{i}=1$, then $y_{j}=0$ whenever $i$ and $j$ differ by \pm 2 or $\pm 3 \bmod n$, and

(b) if $y_{i}=y_{i+1}=1$ then we cannot have $y_{j}=y_{j+1}=1$ when $i$ and $j$ differ by $\pm 5 \bmod n$.

- $C_{16}(1,2,4)$ has no embedding of genus 2

Here the sequence $Y=\left(y_{0}, y_{1} \ldots, y_{15}\right)$ must contain at least eight 1 s, but condition (a) cannot be satisfied when there are more than six $1 \mathrm{~s}$.

- $C_{15}(1,2,4)$ has no embedding of genus 2

In this case there must be at least six $1 \mathrm{~s}$, and up to cyclic rearrangement there is just one possibility satisfying condition (a), namely $Y=(1,1,0,0,0,1,1,0,0,0,1,1,0,0,0)$, but this fails condition (b).

- $C_{14}(1,2,4)$ has no embedding of genus 2

For this value of $n$ there must be at least four 1s, and up to cyclic rearrangement and reversal (corresponding to automorphisms of the graph) we find there are six possibilities for the sequence $Y$ satisfying the condition (a), but two of those fail condition (b), which leaves just the following four: $(1,1,0,0,0,0,1,1,0,0,0,0,0,0),(1,1,0,0,0,0,0,1,1,0,0,0,0,0)$, $(1,1,0,0,0,0,1,0,0,0,1,0,0,0)$ and $(1,1,0,0,0,1,0,0,0,0,1,0,0,0)$.

Each of these has exactly four $1 \mathrm{~s}$, and it follows that $V_{5}=4$. Hence the inequalities $V_{5}+4 n=5 V_{5}+4\left(n-V_{5}\right) \geqslant 3 F_{3} \geqslant 3(2 n-8)=6 n-24$ observed earlier must be equalities, with all expressions being 60 . In particular, there are 20 triangular faces, and all vertices lie in 
either 4 or 5 triangular faces. Then similarly, from $84=4 F-F_{3}=3 F_{3}+4 F_{\ell} \leqslant 2 E=84$ we find that each of the six non-triangular faces must have length 4 .

In all four sub-cases, we may suppose that $\rho_{0}=(-4,-2,-1,1,2,4)$ and then we have $\rho_{1}=(5,3,2,0,-1,-3)$, as previously.

When $Y=(1,1,0,0,0,0,1,1,0,0,0,0,0,0)$ or $(1,1,0,0,0,0,1,0,0,0,1,0,0,0)$, we have $\rho_{6}=(2,4,5,7,8,10)$ or it inverse, but the first is impossible since again it would create a problem with the edge $\{2,4\}$, and therefore $\rho_{6}=(2,10,8,7,5,4)$. It follows that $\rho_{4}$ has the form $(. ., 0,2,6,5, .$.$) , and so has to be (0,2,6,5,3,8)$ or $(0,2,6,5,8,3)$. Now the second of these is impossible since it would give only three triangular faces at vertex 4 , so $\rho_{4}=(0,2,6,5,3,8)$, and moreover, since the faces partially bounded by the 2 -arcs $(0,4,8)$ and $(8,4,3)$ cannot be triangular, the face partially bounded by the 2 -arc $(3,4,5)$ must be triangular. But this implies that edge $\{5,3\}$ is used in triangular faces with the same orientation at vertices 1 and 4; contradiction.

Similarly, when $Y=(1,1,0,0,0,0,0,1,1,0,0,0,0,0)$, we have $\rho_{7}=(3,5,6,8,9,11)$, since using the inverse of this would imply that the edge $\{3,5\}$ is used in triangular faces with the same orientation at vertices 1 and 7 . It then follows that $\rho_{3}$ has the form $(\ldots, 2,1,5,7, \ldots)$, so must be $(2,1,5,7,4,13)$ or $(2,1,5,7,13,4)$. The first of these, however, gives only three triangular faces at vertex 3 , so $\rho_{3}=(2,1,5,7,13,4)$, and moreover, since the faces partially bounded by the 2 -arcs $(13,3,7)$ and $(4,3,13)$ cannot be triangular, the face partially bounded by the 2 -arc $(2,3,4)$ must be triangular. But this forces $\rho_{2}$ to have the form $(\ldots, 3,4,0,1,3, \ldots)$; contradiction.

Thus $Y=(1,1,0,0,0,1,0,0,0,0,1,0,0,0)$. Now we have $\rho_{5}=(9,7,6,4,3,1)$ and so $\rho_{9}=(13,11,10,8,7,5)$, but from these it follows that the unique non-triangular face at vertex 5 is partially bound by the 4 -arc $(13,9,5,1,11)$, so cannot be quadrangular; another contradiction.

- $C_{13}(1,2,4)$ has no embedding of genus 2

In this case the sequence $Y$ contains at least two 1s, and up to cyclic rearrangement and reversal there are exactly ten possibilities satisfying conditions (a) and (b), namely

$$
\begin{array}{lll}
(1,1,0,0,0,0,0,0,0,0,0,0,0), & (1,0,0,0,1,0,0,0,0,0,0,0,0), \\
(1,0,0,0,0,1,0,0,0,0,0,0,0), & (1,1,0,0,0,1,0,0,0,0,0,0,0), \\
(1,0,0,0,0,0,1,0,0,0,0,0,0), & (1,1,0,0,0,0,1,0,0,0,0,0,0), \\
(1,1,0,0,0,0,0,1,0,0,0,0,0), & (1,1,0,0,0,0,1,1,0,0,0,0,0), \\
(1,0,0,0,1,0,0,0,1,0,0,0,0), & (1,1,0,0,0,1,0,0,0,1,0,0,0) .
\end{array}
$$

Note that each of them has at most four 1s.

Next, let $V_{k}$ be the number of vertices incident with exactly $k$ triangular faces. Then $V_{0}+V_{1}+V_{2}+V_{3}+V_{4}+V_{5}=13$, and also $V_{5} \leqslant 4$ (from the ten possibilities for $Y$ ). Moreover, if we let $T$ be the weighted sum $V_{1}+2 V_{2}+3 V_{3}+4 V_{4}+5 V_{5}$, then $T$ is equal to the number of triples $(v, \Delta)$ where $\Delta$ is a triangular face at vertex $v$, and so $T=3 F_{3}$. In particular, $T$ is divisible by 3 , and also $T \geqslant 3 \cdot 18=54$. It is now an easy exercise to show that $\left(V_{0}, V_{1}, V_{2}, V_{3}, V_{4}, V_{5}\right)=(0,0,0,0,11,2),(0,0,0,1,9,3),(0,0,0,2,7,4)$ or $(0,0,1,0,8,4)$. 
In each of these four cases, the weighted sum $T$ is exactly 54 , and so the number of triangular faces is exactly 18 , and then from $54+24=3 F_{3}+4 F_{\ell} \leqslant 2 E=78$ we deduce that the six non-triangular faces must all have length 4.

We can now show that if $y_{i}=1$ then $y_{j}=0$ whenever $j-i \equiv \pm 4$ or $\pm 6 \bmod n$.

For suppose that $y_{0}=y_{4}=1$. We have already assumed that $\rho_{0}=(9,11,12,1,2,4)$. By considering the face bounded by the triple $\{0,2,4\}$, we have also $\rho_{4}=(0,2,3,5,6,8)$. It follows that $\rho_{2}$ is either $(3,4,0,1,6,11)$ or $(3,4,0,1,11,6)$, and in both cases, at vertex 2 we have three non-triangular faces, which must all be quadrangular. When $\rho_{2}=(3,4,0,1,11,6)$, the fourth vertex of the quadrangular face partially bounded by the 2 -arc $(11,1,2)$ cannot be 0 or 12 and so must be 10 , and similarly the quadrangular face partially bounded by the 2 -arc $(3,2,6)$ cannot be 4 or 5 and so must be 7 , but then there is no possibility for the fourth vertex of the third quadrangular face at vertex 2 . The same kind of argument rules out the possibility that $\rho_{2}=(3,4,0,1,6,11)$, by considering the faces containing the edges $\{5,6\}$ and $\{11,12\}$.

Instead, suppose that $y_{0}=y_{6}=1$. In this case, we can take $\rho_{0}=(9,11,12,1,2,4)$ and $\rho_{6}=(10,8,7,5,4,2)$. Then the quadrangular face at vertex 0 must be bounded by the 4-cycle $(9,0,4,8)$, so $\rho_{4}=(0,2,6,5,3,8)$, and similarly, the quadrangular face at vertex 6 must be bounded by the 4 -cycle $(10,6,2,11)$ and then $\rho_{2}=(0,1,3,11,6,4)$. It follows that the fourth vertex of the quadrangular face partially bounded by the 2 -arc $(8,4,3)$ is either 7 or 12 , and so $\rho_{8}$ is either $(7,6,10,12,9,4)$ or $(7,6,10,9,4,12)$, both of which imply that vertex 8 lies in at most three triangular faces. Similarly, $\rho_{11}$ is either $(12,0,9,10,2,7)$ or $(12,0,9,7,10,2)$, and so vertex 11 is incident with at most three triangular faces. Thus $V_{4}+V_{5}$ is at most 11 , and therefore $\left(V_{0}, V_{1}, V_{2}, V_{3}, V_{4}, V_{5}\right)=(0,0,0,2,7,4)$. In particular, every vertex other than 8 and 11 must be incident with at least four triangular faces. This implies that the triple $\{1,2,3\}$ must bound a triangular face at vertex 2 , and similarly, the triple $\{3,4,5\}$ bounds a triangular face at vertex 4 . It follows that $\rho_{5}=(3,4,6,7,9,1)$, and then by considering the edge $\{1,3\}$ we see that the 2 -arc $(3,5,1)$ cannot partially bound a triangular face, so the fourth triangular face at vertex 5 is bounded by $\{5,7,9\}$. But then the fourth vertex of the quadrangular face partially bounded by the 2 -arc $(1,5,9)$ has to be 10 , and that leaves no possibility for $\rho_{1}$.

It follows that there are just two possibilities for $Y$, viz. $(1,1,0,0,0,0,0,0,0,0,0,0,0)$ and $(1,0,0,0,0,1,0,0,0,0,0,0,0)$. Thus exactly two vertices lie in 5 triangular faces, so $\left(V_{0}, V_{1}, V_{2}, V_{3}, V_{4}, V_{5}\right)=(0,0,0,0,11,2)$, and all vertices lie in either 4 or 5 triangular faces.

Now suppose again that $\rho_{0}=(9,11,12,1,2,4)$. Then since vertex 2 lies in exactly four triangular faces, $\rho_{2}=(4,0,1,3,11,6)$, and similarly, $\rho_{11}=(12,0,9,7,2,10)$. But then by considering $\rho_{11}$, we see that the quadrangular face at vertex 2 partially bounded by the 2 -arc $(11,2,3)$ must be bounded by the 3 -arc $(10,11,2,3)$, which is impossible since there is no edge from 3 to 10 .

- $C_{11}(1,2,4)$ has no embedding of genus 2

This is by far the most challenging case, and so left until last. It is another one for which we used a computer, and we give only a partial description of how we handled it.

We assume that it has a genus 2 embedding, which must have 20 faces, with at least 
14 being triangular. Again we consider the sequence $Y=\left(y_{0}, y_{1}, y_{2}, \ldots, y_{n-1}\right)$ as defined above. This time we cannot show that at least one $y_{i}$ is 1 , but condition (a) still holds, namely that if $y_{i}=1$ then $y_{j}=0$ whenever $i$ and $j$ differ by \pm 2 or $\pm 3 \bmod n$.

Up to cyclic rearrangement and reversal we find there are eight possibilities for $Y$. One of them is $(1,1,0,0,0,1,1,0,0,0,0)$, and this can be eliminated using condition (b), but also by showing that if $y_{i}=1$ then $y_{j}=0$ whenever $i$ and $j$ differ by \pm 4 or \pm 5 mod $n$ (when $n=11$ ). Doing that is not as easy to do as it is in the case $n=13$, as there are many more possibilities to check and eliminate.

For example, consider the case where $y_{i}=y_{j}=1$ with $i-j \equiv \pm 5 \bmod 11$. We can deal with that by assuming $\rho_{0}=(-4,-2,-1,1,2,4)=(7,9,10,1,2,4)$, and $\rho_{5}=$ $(1,3,4,6,7,9)$ or its inverse. Choosing $\rho_{5}=(1,3,4,6,7,9)$ creates a problem with the edge $\{7,9\}$, so we must have $\rho_{5}=(1,9,7,6,4,3)$. Then these two rotations at vertices 0 and 5 determine parts of the rotations at the other nine vertices, as follows:

$$
\begin{array}{ll}
\text { Vertex 1: } & \rho_{1}=(2,0,10,5,3,8) \text { or }(2,0,10,8,5,3) ; \\
\text { Vertex 2: } & \rho_{2}=(4,0,1, a, b, c) \quad \text { where }\{a, b, c\}=\{3,6,9\} ; \\
\text { Vertex 3: } & \rho_{3}=(1,5,4, d, e, f) \quad \text { where }\{d, e, f\}=\{2,7,10\} ; \\
\text { Vertex 4: } & \rho_{4}=(3,5,6,0,2,8) \text { or }(3,5,6,8,0,2) ; \\
\text { Vertex 6: } & \rho_{6}=(4,5,7, g, j, k) \text { where }\{g, j, k\}=\{2,8,10\} ; \\
\text { Vertex 7: } & \rho_{7}=(6,5,9,0,3,8) \text { or }(6,5,9,0,8,3) ; \\
\text { Vertex 8: } & \rho_{8}=(1, p, q, r, s, t) \text { where }\{p, q, r, s, t\}=\{4,6,7,9,10\} ; \\
\text { Vertex 9: } & \rho_{9}=(10,0,7,5,2,8) \text { or }(10,0,7,5,8,2) ; \\
\text { Vertex 10: } & \rho_{10}=(1,0,9, x, y, z) \text { where }\{x, y, z\}=\{3,6,8\} .
\end{array}
$$

Hence we have 2 possibilities for the rotations at each of the vertices 1, 4, 7 and 9, and 6 possibilities for the rotations at each of the vertices 2, 3, 6 and 10, and 120 possibilities for the rotation at vertex 8 . These combine to give a total of $2^{4} \cdot 6^{4} \cdot 120=2488320$. Clearly further observations can be made to reduce this number, but even so, it is a small enough number that all possibilities can easily be checked by computer. We carried out a 35-minute computation using MAGMA on a laptop, and found that the smallest genus achievable from such choices of rotations is 3 . In particular, genus 2 is impossible when $y_{0}=y_{5}=1$.

Similarly, when $y_{0}=y_{4}=1$ and $\rho_{0}=(7,9,10,1,2,4)$, the total number of possibilities for the rotation system is $2 \cdot 6^{6} \cdot 24^{2}=53747712$, and a longer (13-hour) computation shows that the smallest genus achievable is again 3 .

Hence if $y_{i}=1$ then $y_{j}=0$ whenever $i$ and $j$ differ by $\pm 2, \pm 3, \pm 4$ or $\pm 5 \bmod n$. It follows that at most two terms in the sequence $Y$ can be 1 , and if there are two, they must be in adjacent positions, so $Y=(1,1,0,0,0,0,0,0,0,0,0),(1,0,0,0,0,0,0,0,0,0,0)$ or $(0,0,0,0,0,0,0,0,0,0,0)$.

The same kind of computational approach as described immediately above can also be adapted to show that we cannot have $y_{0}=y_{1}=1$. This takes rather longer (73 hours by computer), but again shows that genus 3 is the smallest possible, and so it eliminates the first of the remaining three possibilities for $Y$. 
Next, we consider the second possibility, namely $Y=(1,0,0,0,0,0,0,0,0,0,0)$. Again suppose that vertex 0 lies in five triangular faces, and $\rho_{0}=(7,9,10,1,2,4)$, and again let $V_{k}$ be the number of vertices incident with exactly $k$ triangular faces. Then $V_{5}=1$, and the weighted sum $T=V_{1}+2 V_{2}+3 V_{3}+4 V_{4}+5 V_{5}$ is at least $3 \cdot 14=42$, and we find that $\left(V_{0}, V_{1}, V_{2}, V_{3}, V_{4}, V_{5}\right)=(0,0,0,0,10,1),(0,0,0,3,7,1),(0,0,1,1,8,1)$ or $(0,1,0,0,9,1)$.

In the first of these four cases, all vertices except 0 (say) lie in exactly four triangular faces, and this makes it easy to eliminate. But we can do that another way, more helpfully. It is easy to prove that if vertex 2 lies in four triangular faces, then vertex 3 cannot, and similarly, if vertex 9 lies in four triangular faces, then vertex 8 cannot. Hence at least two vertices lie in three or fewer triangular faces, and this rules out the first and fourth of the above possibilities for $\left(V_{0}, V_{1}, V_{2}, V_{3}, V_{4}, V_{5}\right)$, leaving only $(0,0,0,3,7,1)$ and $(0,0,1,1,8,1)$.

Hence in particular, the number of vertices lying in at most three triangular faces is $V_{0}+V_{1}+V_{2}+V_{3}$, which is either 3 or 2 , and moreover, these vertices include at least one of vertices 2 and 3 , and at least one of vertices 8 and 9 . It follows that at least one of the two vertices 4 and 7 must lie in four triangular faces.

Given that $\rho_{0}=(7,9,10,1,2,4)$, we find there are just seven possibilities for $\rho_{4}$, namely $(0,2,3,5,6,8),(0,2,3,5,8,6),(0,2,3,8,6,5),(0,2,6,5,3,8),(0,2,6,8,3,5),(0,2,6,8,5,3)$ and $(0,2,8,6,5,3)$. The first, third and fourth of these cases can be eliminated with the help of a computer, and in the second, fifth and seventh cases, it is easy to prove that at least two of the vertices $1,2,3$ and 5 lie in at most three triangular faces, while in the sixth case, either the latter happens, or vertex 3 lies in at most two triangular faces. Similarly (by replacing elements of $\mathbb{Z}_{11}$ by their negatives), we find that if vertex 7 lies in four triangular faces, then either vertex 8 lies in at most two triangular faces, or at least two of the vertices 6,8,9 and 10 lie in at most three triangular faces. Both of these are impossible since $\left(V_{0}, V_{1}, V_{2}, V_{3}, V_{4}, V_{5}\right)=(0,0,0,3,7,1)$ or $(0,0,1,1,8,1)$, so vertex 7 cannot lie in four triangular faces.

Now we have at least three vertices lying in at most three triangular faces, namely vertex 7 , one of 2 and 3 , and one of 8 and 9 . Thus $\left(V_{0}, V_{1}, V_{2}, V_{3}, V_{4}, V_{5}\right)=(0,0,0,3,7,1)$. In particular, $V_{0}+V_{1}+V_{2}=0$, so vertex 3 cannot lie in at most two triangular faces, and therefore at least two of the vertices 1, 2, 3 and 5 lie in at most three triangular faces. This is one too many; contradiction.

Finally, we suppose that $Y=(0,0,0,0,0,0,0,0,0,0,0)$. Here $V_{5}=0$, and it is easy to see that $\left(V_{0}, V_{1}, V_{2}, V_{3}, V_{4}, V_{5}\right)=(0,0,0,2,9,0)$ or $(0,0,1,0,10,0)$.

In both of these two cases, the weighted sum $T$ is 42 , so there are 14 triangular faces, and then from $42+24=3 F_{3}+4 F_{\ell} \leqslant 2 E=66$ we find that the six non-triangular faces all have length 4 . Moreover, since $\left(V_{0}, V_{1}, V_{2}, V_{3}, V_{4}, V_{5}\right)=(0,0,0,2,9,0)$ or $(0,0,1,0,10,0)$, we know there are at most two vertices that are not in four triangular faces, say $u$ and $v$. In particular, since $|u-v|$ is at most 5 , we can suppose that $v=u+j$ where $1 \leqslant j \leqslant 5$, and then $u=v+k$ where $6 \leqslant k \leqslant 10$. It follows that each of the five vertices $v+1, v+2$, $v+3, v+4$ and $v+5$ lies in four triangular faces, and without loss of generality we may take $v=8$, and assume that these five vertices are $9,10,0,1$ and 2 .

Under this assumption, an easy exercise shows that up to reflection there are just eight possibilities for the rotation $\rho_{0}$, namely $(1,2,4,7,9,10),(1,2,4,9,7,10),(1,2,4,10,9,7)$, 
$(1,2,9,7,4,10),(1,4,2,7,9,10),(1,4,2,9,7,10),(1,7,4,2,9,10)$ and $(1,7,9,2,4,10)$, which we will call $\pi_{1}$ to $\pi_{8}$.

In the first case, which involves the rotation we took at vertex 0 when we assumed 0 lay in five triangular faces, one of the two non-triangular faces is partially bounded by the 2 -arc $(7,0,4)$, and the other is bounded by a 4-cycle of the form $(u, 0, v, w)$ where $u$ and $v$ are adjacent in the graph. In this situation, we can change the rotations at vertices $u$ and $v$ so that the edge $\{u, v\}$ becomes a diagonal, splitting the latter face into two faces of length 3 , while the two faces that previously contained the edge $\{u, v\}$ are merged into a single face. This achieves a different embedding with the same genus 2 , but with five triangular faces at vertex 0 , which we know is impossible. Hence $\rho_{0}$ is one of $\pi_{2}$ to $\pi_{8}$.

The same arguments show that the only possibilities for the rotation at each of the vertices 9, 10, 1 and 2 are the analogues of $\pi_{2}$ to $\pi_{8}$, obtained by adding respectively 9 , 10,1 or $2(\bmod 11)$ to the points of each $\pi_{i}$, and their inverses.

Also we note that under the automorphism of the circulant taking each $v$ to $-v$, the possibilities $\pi_{2}=(1,2,4,9,7,10)$ and $\pi_{5}=(1,4,2,7,9,10)$ are equivalent to the inverse of each other, as are $\pi_{4}=(1,2,9,7,4,10)$ and $\pi_{7}=(1,7,4,2,9,10)$, while $\pi_{3}=(1,2,4,10,9,7)$ is 'self-equivalent', and each of $\pi_{6}=(1,4,2,9,7,10)$ and $\pi_{8}=$ $(1,7,9,2,4,10)$ is equivalent to its own inverse. Hence we may assume that $\rho_{0}$ is $\pi_{2}, \pi_{3}$, $\pi_{4}, \pi_{6}$ or $\pi_{8}$.

Now suppose $\rho_{0}$ is $\pi_{2}=(1,2,4,9,7,10)$. Then the two non-triangular faces at 0 are partially bounded by the 2 -arcs $(9,0,4)$ and $(10,0,7)$, while the four triangular faces are bounded by the 3 -cycles $(2,0,1),(4,0,2),(7,0,9)$ and $(1,0,10)$. At vertex 2 , the rotation must have the form $(. ., 4,0,1, .$.$) , and by looking at the analogues of \pi_{2}$ to $\pi_{8}$, we find there is just one possibility, namely $(3,9,6,4,0,1)$, the analogue of $\pi_{7}$. In particular, the four triangular faces at vertex 2 are bounded by the 3 -cycles $(1,2,0),(0,2,4)$, $(4,2,6)$ and $(3,2,1)$. But then it follows that the rotation at vertex 1 must have the form $(. ., 3,2,0,10, .$.$) , and therefore cannot be the analogue of any of \pi_{2}$ to $\pi_{8}$; contradiction.

Instead, suppose $\rho_{0}$ is $\pi_{3}=(1,2,4,10,9,7)$. Then the two non-triangular faces are partially bounded by the 2 -arcs $(10,0,4)$ and $(1,0,7)$, while other four triangular faces at vertex 0 are triangular. Again $\rho_{2}$ must be $(3,9,6,4,0,1)$, and it follows that $\rho_{4}$ has the form $(. ., 6,2,0, .$.$) . Similarly, at vertex 9$ the rotation must have the form $(. ., 7,0,10, .$.$) ,$ and we find there is just one possibility, namely $(7,0,10,8,2,5)$, the analogue of $\pi_{4}^{-1}$, and then $\rho_{7}$ has the form $(. ., 0,9,5, .$.$) . Now at vertex 1$, the rotation has the form $(. ., 3,2,0, .$.$) , so must be (3,2,0,8,10,5)$ or $(3,2,0,5,8,10)$, analogous to $\pi_{2}^{-1}$ or $\pi_{4}^{-1}$. Hence the quadrangular face containing the edges $\{0,1\}$ and $\{0,7\}$ must be bounded by either $(1,0,7,8)$ or $(1,0,7,5)$, but the latter is inconsistent with the rotation at vertex 7 , and it follows that $\rho_{1}=(3,2,0,8,10,5)$. In particular, $(1,8,10)$ bounds a triangular face, and therefore at vertex 10 , the rotation has the form $(. ., 1,8,9,0, .$.$) . It follows that$ $\rho_{10}=(0,6,3,1,8,9)$, analogous to $\pi_{7}$, but this inconsistent with the rotation at vertex 4 ; contradiction.

Similar arguments rule out the possibilities $\pi_{4}, \pi_{6}$ and $\pi_{8}$ for $\rho_{0}$. (When $\rho_{0}=\pi_{6}$, we find three candidates for $\rho_{1}$, viz. the analogues of $\pi_{3}, \pi_{5}^{-1}$ and $\pi_{7}^{-1}$, but all are impossible.)

Thus we find no possibility for a rotation system, and it follows that $C_{11}(1,2,4)$ has 
no embedding of genus 2. This completes the proof of Theorems 2 and 3.

\section{Acknowledgements}

Much of the work described in this paper was carried out when the second author visited the University of Auckland in July and August 2012, with the award of a 'Northern Hemisphere Summer Scholarship' from its Faculty of Science. The first author is grateful to the N.Z. Marsden Fund for its support (grant UOA 1015), and acknowledges the use of the Magma system [3] for computational experiments and verification of a number of discoveries announced in this paper. The second author would like to thank Luis Martínez and Gustavo Fernández for their help and support throughout and following the application process that led to his visiting the University of Auckland, and is also grateful to the Mathematics Department and Science Faculty at the University of Auckland and the Faculty of Science and Technology at the University of the Basque Country for their support.

\section{References}

[1] J. Battle, F. Harary, Y. Kodama and J.W.T. Youngs, Additivity of the genus of a graph, Bull. Amer. Math. Soc. 68:565-568, 1962.

[2] N.L. Biggs and A.T. White, Permutation Groups and Combinatorial Structures, London Math. Soc. Lecture Note Series, vol. 33. Cambridge University Press, 1979.

[3] W. Bosma, J. Cannon and C. Playoust, The Magma Algebra System I: The User Language, J. Symbolic Computation 24:235-265, 1997.

[4] M.G. Brin and C.C. Squier, On the genus of $\mathbb{Z}_{3} \times \mathbb{Z}_{3} \times \mathbb{Z}_{3}$, European J. Combin. 9:431-443, 1988.

[5] I.S. Filotti, G.L. Miller, and J. Reif, On determining the genus of a graph in $O\left(v^{O(g)}\right)$ steps, Proceedings 11th Annual ACM Symp. Theory of Computing, pp. 27-37, 1979.

[6] J. Gross and T.W. Tucker, Topological Graph Theory, John Wiley \& Sons, Inc., New York, 1987 (and reprinted by Dover Publications, 2001).

[7] C. Heuberger, On planarity and colorability of circulant graphs, Discrete Math. 268:153-169, 2003.

[8] B. Mohar, T. Pisanski, M. Škoviera and A.T. White, The cartesian product of three triangles can be embedded into a surface of genus 7, Discrete Math. 56:87-89, 1985.

[9] G. Ringel, Das Geschlecht des vollständigen paaren Graphen, Abh. Math. Sem. Univ. Hamburg 28:139-150, 1965.

[10] G. Ringel and J.W.T. Youngs, Solution of the Heawood Map-Coloring Problem, Proc. Nat. Acad. Sci. USA 60:438-445, 1968.

[11] M. Škoviera and R. Nedela, The maximum genus of vertex-transitive graphs, Discrete Math. 78:179-186, 1989. 
[12] J.E. Strapasson, S.I.R. Costa and M.M.S. Alves, On genus of circulant graphs, 2010. arXiv: $1004.0244 \mathrm{v} 1$

[13] C. Thomassen, The graph genus problem is NP-complete, J. Algorithms 10:568-576, 1989.

[14] C. Thomassen, Tilings of the torus and the Klein bottle and vertex-transitive graphs on a fixed surface, Trans. Amer. Math. Soc. 323:605-635, 1991. 